\title{
Discussion of Steinmetz and Slichter Papers.
}

B. G. Lamme:-In the paper presented before the American Institute of Electrical Engineers in September, 1902, the speaker called attention to the fact that there were but two types of single-phase alternating-current motors having suitable characteristics for railway service; viz., that called the "Series Type," and the "Repulsion Type." Both of these motors have armatures like direct-curi ent motors, with commutators and brush holders. Attention was called to the fact that both motors have suitable characteristics for railway service, as both automatically give variable-speed characteristics with changes in load. That paper primarily described a single-phase railway system, and the motor formed but an element in the general system. It was a very general opinion at that time that the success of the commutator type of motor for large sizes was doubtful, and the sparking feature was considered a fundamental source of trouble. It was generally conceded that if a motor with series characteristics could be made to operate successfully, it would be a great step in advance in the railway field.

Since that time single-phase railway systems have been more fully developed. Practically no departures from the general system then indicated have been furnished, and the types of motors developed have been along the lines of the two motors indicated in that paper.

Up to the present time the only suitable motors suggested for this work have been of the commutator type, and have been those having series characteristics. The speaker has suggested that all these motors can be considered broadly under the one class of series motors, as they all have the series characteristics of the direct-current series motor. The speaker further suggested that they be subdivided into the "Straight-Series" type and the "Transformer-Series" type. The transformerseries could also be arranged in two classes; viz., one in which the armature or field is supplied by an external transformer, and one in which the transformer is placed in the motor itself; this latter is the repulsion type of motor.

Figs. 1, 2, and 3 illustrate the three classes. Fig. 1 being the straight-series, Fig. 2 the transformer-series, and Fig. 3 the repulsion motor. Fig. 2 would be considered as a true series motor, although the armature and field are not directly in series, vet most of the characteristics described as belonging in the repulsion motor apply directly to the transformer motor shown in the figure. Comparing the relations of these motors; viz., the straight-series and the repulsion motor, we will first take up the straight-series.

In this motor, if properly designed, two pressures can be considered; viz., that across the field circuit, and that across the armature circuit. The armature pressure can be made practically non-inductive so that the input of the armature will represent practically true energy. The pressure across the field 
is practically at right angles to the armature pressure, and represents very closely the wattless component supplied by the motor. The resultant of these two pressures will then be the line pressure. The power-factor of the motor when running is represented practically by the pressure across the armature winding, increased slightly by the losses in the field-core and winding. Therefore, for high power-factors it is important that the pressure across the armature circuit be made as high as possible, relatively to the applied pressure, and that across the field as low as possible.

There are three ways in which to increase the pressure across the armature; viz., by increase in speed, by increase in the number of wires in series on the armature, and by increase in flux through the armature.

By increase in speed and increase of the wires in series, the armature pressure will be increased without affecting the field
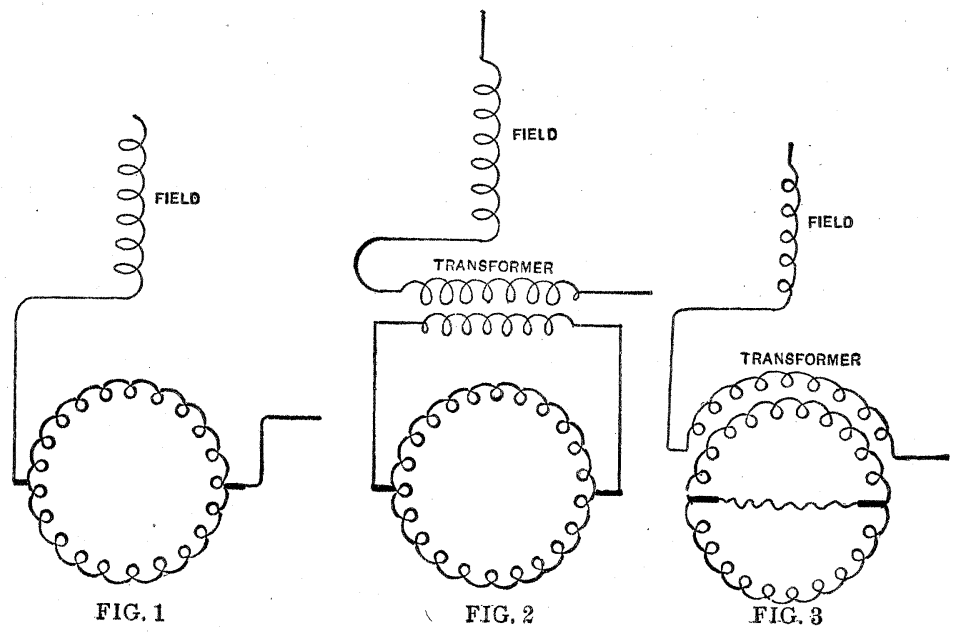

pressure, and therefore the ratio of the armature pressure to the line pressure is increased. Increasing the flux in the armature also increases the flux in the magnetizing-coil in the field, and the pressures of both are increased. Therefore this increase does not improve the power-factor of the machine.

Instead of increasing the armature pressure, the pressure across the field winding may be decreased; this can be done in two ways; viz., by reducing the turns in the field coil, or by reducing flux through the coil. Reducing the flux through the field reduces the flux in the armature winding also, and therefore mepresents no gain; reduction in field-turns, therefore, is the feasible means of reducing the field pressure. Reduction in field-turns can be accomplished in two ways; viz., by decreasing the effective length of air-gap in the motor, and by increasing the cross-section of gap. By making the gap very small the pres- 
sure across the field could be made very small compared with the line pressure, and extremely high power-factors could be obtained, whether the motor is of the straight-series or the repulsion type. Also by increasing the section of the air-gap the turns of the field can be decreased with a given total flux through the coil, and the power-factor can thus be very considerably increased. The first method, viz., decrease in gap, is limited by practical conditions which have been determined from long experience with direct-current work. It should be borne in mind when published descriptions of such motors are given that the results, as regards power-factor, generally depend upon data which are not given in the description,-such as the magnetic dimensions of the armature and field, the length of gap, etc. Therefore, a machine may be described as showing an extremely high power-factor, which may in practice not be a commercial machine, from the standpoint of American railway experience. Increasing the section of air-gap without decreasing the length of gap also improves the power-factor, but makes a larger and heavier machine, as a rule.

Both these modifications reduce the ampere-turns in the field. The direction of the improvement in the armature was shown to be in increased armature ampere-turns with a given speed. It therefore follows that almost any result desired can be obtained as regards power-factor by increasing the armature ampere-turns, and decreasing the field or exciting ampere-turns. Reference will be made to this point in considering the repulsion motor.

It should be noted that in all these motors there should be but little saturation in the magnetic circuit and but few ampereturns expended in saturation of the iron under normal conditions. This consequent low saturation in such motors leads to certain characteristics in the torque curves which have been cited this evening as an indication of superiority of alternating-current motors over direct-current motors; namely, a torque increasing approximately as the square of the current. In fact, this superiority of torque should be charged to the low flux-density of the motor rather than to the alternating current. If direct-current motors were worked normally at as low density as the alternatingcurrent motor, then the direct-current motor would show better torque characteristics, and would be comparable with the alternating-current motor. This claim for a better torque in the alternating-current motor compared with the direct-current motor seems to be making a virtue of a necessity.

It is evident from what has been said that the power-factor of the straight-series motor can be made anything desired, it being a question of proportion between armature and field, length of air-gap, amount of material used, etc. In practice a compromise would naturally be made among the various characteristics, and a slight reduction in power-factor is probably of less importance than a corresponding reduction in size and weight. Also large 
clearance is probably of more importance than an extremely high power-factor at normal load. In practice it will be found that the armatures of such motors have a large number of ampereturns compared with the fields, in order to obtain comparatively high power-factors with large air-gaps. The number of poles need not be made such that the product of the poles by the normal speed represents the frequency of the supply circuit; good series motors can be made, and have been made, in which the number of poles were very much larger or much smaller than represented by this relation.

Taking up next the transformer type of motors-Fig. 2 ; the field is in series with the primary of the transformer, the secondary of which is connected to the terminals of the motor. The speaker would call this a true series motor, although it is not a straightseries motor. In this motor the pressure across the armature can be made practically non-inductive and the pressure across the primary of the transformer will be practically non-inductive. The voltage across the field winding will have practically $90^{\circ}$ phase relation to that across the primary of the transformer, and the magnetic field, set up by the field winding, will have a $90^{\circ}$ relation in time to the magnetic field in the transformer, as in the repulsion motor. In this motor the voltage across the transformer will be highest at light loads and will decrease with load until zero speed is reached. At start there is lowest flux in the transformer and highest flux in the field winding. Such a motor will have speed-torque characteristics very similar to those of a straight-series motor, except as affected by the actions taking place in the transformer itself. If the transformer possesses no reactance, then at start the current in the armature should be the same as if connected as straight-series motor, and the conditions of torque at start should be the same. If the transformer has reactance, then at start the current in the armature will not be quite equal to the current which the armature will receive if coupled as a straight-series motor, assuming the transformer to have a 1 to 1 ratio. Neither will the armature current be exactly in phase with the field current; therefore the starting torque of a motor connected in this way will be slightly less than the torque of the same motor if connected in straight-series. This is on the assumption that the transformer is one proportioned for small reactance; but if the primary and secondary windings of the transformer should be on separate cores with air-gap between, then the reactances of the windings are considerably greater than in the above case. Therefore, we should expect a motor with such a transformer to give still lower torque than the straight-series with the same current supplied from the line.

In a repulsion motor the transformer is combined with the motor itself and the primary and secondary windings are upon different cores with an air-gap between. The starting conditions of such a motor as indicated above should be poorer than 
the straight-series motor, or for the same starting torque somewhat greater apparent energy should be required. It stands to reason that applying the current directly to the armature winding should give greater ampere-turns and better phase relations than generating this current in a secondary circuit, and not under ideal transformer conditions. The tests which have been made, as well as the results shown in the curves of the papers given to-night indicate this. It is to be noted that the torque curve is not the same shape near the zero-speed point as the torque curve of the series motor.

Series motors and repulsion motors may be indicated in the simple form shown in Figs. 4 and 5 . In the diagrams of the repulsion motor (Fig. 5), two field-poles, $F F$, are shown, and two transformer-poles, $T T$. To obtain high power-factors on such a motor the ampere-turns in $T$ must be very much greater than in $F$; which means that the ampere-turns in the secondary or arma-

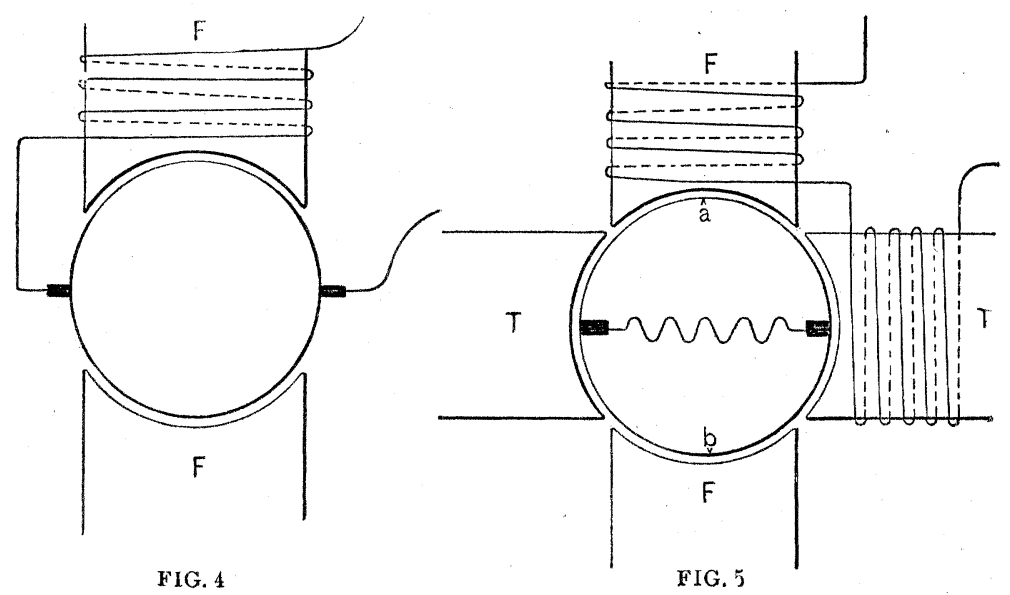

ture are much greater than in the exciting field, as in the series motor. The high power-factor obtained with these motors is therefore due principally to the small ampere-turns in the field and the small pressure across the field.

For instance, with brushes set at an angle of $16^{\circ}$, from the primary or resultant field, the ratio of armature to exciting fieldturns would be almost 5 to 1 , a ratio which will also permit of extremely high power-factors in well-designed straight-series motors over wide ranges of speed. To this feature should be credited the good power-factors claimed for the repulsion motor. In either the series of repulsion type of motors, high powerfactors, especially at low speeds, are directly dependent upon this fact of high ratio of armature to field, and with a high ratio high power-factors should be obtained without crediting the result to leading currents in the armature. In the diagram of the repul- 
sion motors the line current indicated flows through both the field winding and the transformer winding. The primary current sets up a magnetic field in the exciting windings in phase with the line current. If it also set up a field in the transformer in phase with the line current, then the electromotive force generated in the armature winding due to rotation would have a $90^{\circ}$ relation to the electromotive force set up by the transformer, and a correcting or magnetizing current would flow. This flow is in such direction that it corrects the relation between the two press ures in the armature by shifting the transformer magnetism one-quarter phase later than the exciting field magnetism. This armature corrective current may thus be considered as magnetizing the transformer, making the primary input to the transformer practically non-inductive; but this magnetizing or correcting current may be considered as flowing in a circuit at right angles to the field magnetic circuit, and having practically no effect on the field circuit. Therefore, as a rough approximation the exciting field may be considered to represent the wattless component of the input, and the transformer field the energy component, as in the series motor. As to the statement that the magnetizing current in the armature reduces the wattless component of the exciting field, the speaker does not accept it broadly. If this component is reduced, then another component of practically equal value is introduced somewhere else, for the powerfactors obtained with such motors can be accounted for by the high ampere-turns in the armature winding, compared with the field or exciting ampere-turns. If the armature current improves the power-factor by diminishing the magnetizing or exciting field, then the curves in Figs. 1 and 4 of Mr. Slichter's paper should show it. The speaker has gone over both sets of curves calculating the wattless components from the power-factors. From this and other data in these curves, he finds that beginning near synchronous speed the wattless component in the motor goes up slightly faster than would be represented by the field excitation, assuming it to be entirely wattless. Therefore, according to these curves, the power-factors at lower speeds are not quite as good as would be obtained by a field entirely inductive and the armature entirely non-inductive, in a straight-series motor. These calculations are rather approximate, as the curves do not check at all well with each other. For instance, the output of the motor as represented by the input multiplied by the powerfactor and by the efficiency, does not check with the output as represented by the product of speed by torque, in either set of curves, the discrepancies being as high as 10 per cent. In Fig. 4, for instance, either the torque or the speed is too high for the lower speeds. Checking back on this curve, using either the speed and torque or the power-factor and efficiency for determining the output, the speaker finds that the wattless component in the motor at 190 revolutions is approximately 20 per cent higher than it would be if the field excitation alone were wattless, 
assuming at 440 revolutions the wattless component is represented purely by field excitation; that is, from 440 to 190 revolutions the wattless component is increased 20 per cent over that which would be represented by field excitation alone. This indicates that not only should the field excitation be considered as practically wattless, but that in addition there is a wattless component due to reactances in the armature windings.

The armature current can be split into two components, one of which is partly magnetizing and represents no torque. The other component is in phase with the field magnetism and therefore represents torque. The magnetizing or wattless element may be comparatively small, as the number of turns in the armature is relatively large, but the armature thus carries at times a slightly larger current than the straight-series motor.

A further inspection of the diagram (Fig. 5) indicates how the power-factor of the motor can be made very high at synchronous speed. At all speeds the pressure generated in the armature due to rotation in the field of $F$ is practically equal to the pressure generated by the transformer $T$, thus making zero pressure across the terminals. But also at synchronous speed the pressure generated by the exciting field acting as a transformer, between the points $a b$, will be practically equal to the pressure generated in the winding by rotation of the winding in the transformer field. Therefore across $a b$ the pressure is practically zero with these conditions, but the frequency remains the same as that in the field. If now the magnetizing current be supplied across the points $a b$, then the required ampere-turns for magnetizing the motor can be supplied at practically zero pressure, and the turns of the external magnetizing field can be omitted. Therefore, under this condition the wattless component is practically zero and the power-factor becomes practically 100 per cent. This is the method of excitation used on certain European single-phase motors in which high power-factors are claimed for full-load running. But this method of excitation does not improve conditions at start, as the same excitation will be required at standstill, whether the excitation be supplied to the armature or to the field. Therefore this method of excitation does not help the motor at that condition of load which is the severest on the generating and transmission system. It has the advantage of omitting the field-exciting winding, but has the great disadvantage of requiring a double set of brushes on the commutator, with but half the distance between the brushes found in the straight-series or the ordinary repulsion motor. The speaker does not believe that such methods of compensation are of sufficient advantage to overcome the complications attendant upon them.

At zero speed, both the straight-series and the repulsion motors have low power-factors, and with equal losses in the motors the repulsion should have slightly lower power-factor than the series. This question of power-factor at start is largely a question of internal losses in the motor at rest, and the repul- 
sion motor in individual cases may show higher than the series motor, because it may be designed with higher internal losses. The real measure of effectiveness is not the $p$,wer-fact or at start, but the apparent input or kilovolt-amperes at start required for a given starting torque. With equally good designs of motors, the speaker's experience is that the kilovolt-amperes will be found to be considerably less with the straight-series than with the repulsion motor, due to the fact that the current is fed directly into the armature and not by transformer action, and therefore the conditions of phase-relation and amount of current in the armature windings are more favorable. Therefore it follows that in order to have the same kilovolt-ampere input for the same starting torque, the repulsion motor should have a smaller length of air-gap than the corresponding straight-series motor, or should have a greater section of air-gap, which means greater weight of motor. This is one of the conditions which has led

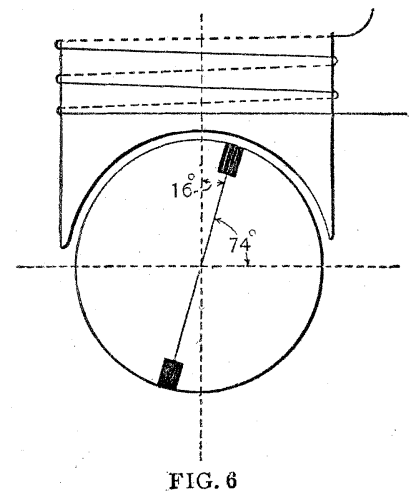

FIG. 6

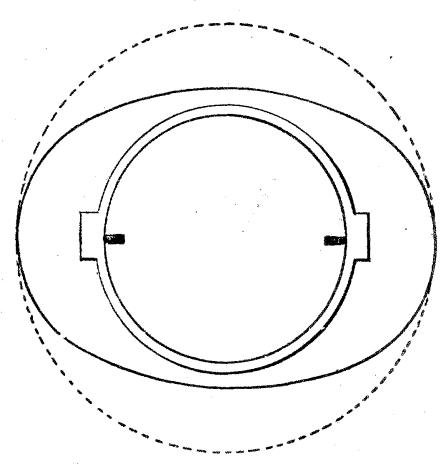

FIG. 7

the speaker to the advocacy of the series motor rather than the repulsion motor, as he has considered this condition of starting of more importance than running; although he is satisfied that many of the running conditions of a well-designed series motor will be found in practice to be superior to those of an equally well-designed repulsion motor.

Referring again to Fig. 5, it will be noted that two fields are set up in such a motor, and that at synchronous speed these two fields are equal. In the straight-series motor there is but one field set up, the other being omitted. It is evident that the straight-series motor with the current supplied directly to the brushes can have a smaller section in sertain parts of the magnetic circuit than is required for the repulsion motor, and that therefore the weight of material would be less, and the external dimensions can beless. In Fig. 7 the heavy line represents outlines of series and the dotted line those of repulsion motor; therefore, it follows that for equally good designs and same fre- 
quency, the straight-series motor should be more compact, and should weigh less than the repulsion motor. It is reasonable to expect this, as the repulsion motor contains a transformer in addition to the other parts found in the straight-series motor. Furthermore, the transformer found in such a motor is one with an air-gap, and with the windings on two separate elements, and therefore cannot be so well proportioned as a separate transformer could be. Also, there is a transformer for each motor, and in a 4-motor railway equipment, for instance, there would be four transformers of smaller size against one larger transformer used with the series motor, this larger transformer having a closed magnetic circuit, and of a highly efficient design compared with the transformers in the motors themselves.

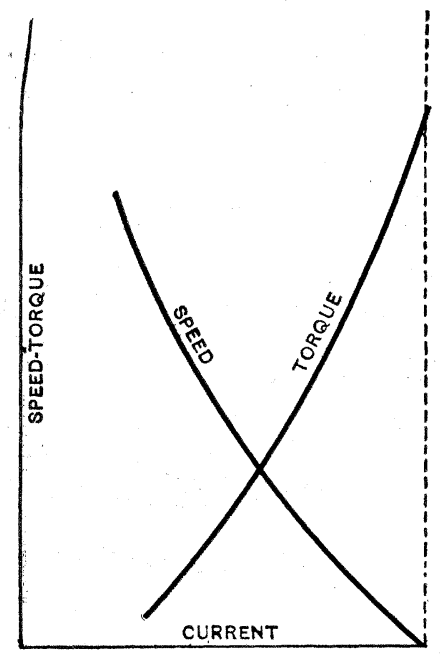

Straight. Series Motor

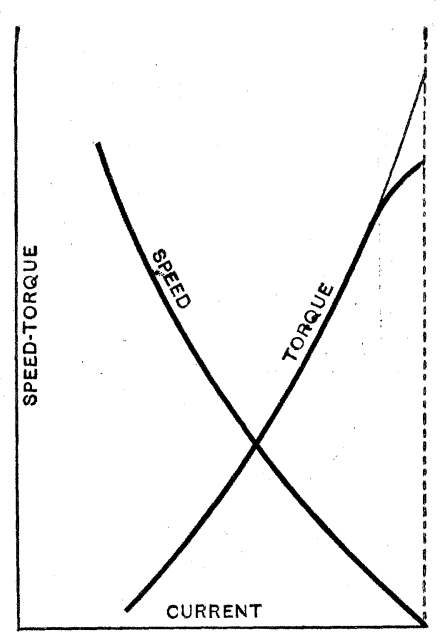

Repulsion Motor

A further point should be taken up in the comparison of these motors; viz., the current in the coil short circuited by the brushes. This coil is a secondary to the field and the current in it is necessarily greatest at the period of strongest field. Therefore, this current will be greatest at the time of starting. If the repulsion motor and the straight series motor have the same field strength at start, then the short-circuited current should be the same in each. But as the current is fed into the armature in the repulsion motor through transformer action, it will, as a rule, be found that the starting field strength of such a motor is slightly greater and the starting armature strength slightly less for a given torque than is found in the straight-series motor having same ratio of armature to field windings. Therefore the short-circuit current at start will be somewhat larger for the repulsion motor than for the corresponding straight-series motor. 
This short-circuit current may be somewhat less near full speed than in the straight-series motor, but it is not the full-speed condition which is the serious one. The short-circuit current at start is one of the most serious conditions which confronts us in alternating-current motors, and is also of great importance where there is any considerable operation on low speeds. The speaker advocates a type which he considers gives the easiest condition in this regard. This short-circuiting cannot be entirely avoided in any of the motors brought out without adopting abnormal and questionable constructions, although devices ike narrow brushes, sandwich windings, etc., have been proposed. In certain foreign motors the brushes used are so narrow that they cover practically the width of one commtuator-bar. As such motors are generally built with a very large number of bars, the brushes used are extremely narrow, being approximately 0.2 inch thick at the tip. This will undoubtedly lessen the short circuiting, but simply transfers trouble to another point; a brush 0.2 inch thick is not practicable for commercial railway service; at high speeds, with only a moderately rough commutator, such brushes will be liable to chip and break; further, the brush on a street-car motor should bridge at least two bars to give good, smooth, brush operation; in practice, a 0.5 inch brush on motors of $100 \mathrm{hp}$. should be used.

The sandwich winding, which consists of two or more windings side by side, will prevent short circuiting at the brushes, but is only another way of transferring trouble to another point; it has been found in practice that it is difficult to run a sandwich winding without trouble at the commutator with direct current without a tendency to blackening and pitting the commutator, and with alternacing current this tendency to pitting and burning of the bars would be equally great.

As a rule, there is little difference between the operation of repulsion and straight-series motors as regards sparking, except that the repulsion motors generally have greater current in the short-circuited coil near zero speed, and therefore show greater tendency to heat and spark. At or near synchronous speed, there appears to be very little difference in the commutation, although the speaker has never given the repulsion motor the same test of long-continued service as he has in the case of the series motor. These series motors have never shown any tendency to give trouble on the commutator, and on an exhibition car equipped with four 100-hp. motors, the commutators have never been sandpapered since the equipment has been put into service. This exhibition car is used principally for showing the accelerating properties of the motors; therefore, the speaker does not hesitate to say that the commutation of the straightseries motor will prove to be equal to that of the direct-current motor. Wide brushes are used with it, such as have been used in street-railway motors.

It is well known that with large direct-current motors, espe- 
cially when operated at very high speeds, there is a tendency to flash across the commutator, or to the frame of the motor, if the field circuit be opened for a period long enough to allow magnetism to drop to zero, and then the field be closed again. In this case there is a rush of current before the field has had time to build up, and this rush of current, together with field distortion, may cause serious flashing. In the alternating-current motor, whether of the straight-series or the repulsion type, this tendency should be entirely absent. In the straight-series motor the magnetism falls to zero once in each alternation, and therefore if this tendency existed, flashing would occur continuously. Furthermore, a properly designed straight-series motor can be short circuited across the brushes without injury to the motor, and can continue to operate in this way; therefore, if the machine can be short circuited in this way, there is evidently no tendency to maintain an arc.

Returning to the subject of power-factors it should be noted that high power-factors are very frequently found in motors of low or only moderately good efficiency. This low efficiency to a slight extent explains the high power-factor in some motors, both polyphase and single-phase. Low efficiency means higher true energy expended, and with a given wattless component it means higher power-factor. It is the old problem of increasing the power-factor by wasting energy in a circuit instead of reducing the wattless component. The power-factor of any alternatingcurrent motor can be very considerably increased by putting resistance in series with it. Instead of this resistance the internal losses of the motor may be made higher, which will accomplish the same results. The motor will therefore appear to have a higher power-factor than it really deserves, if efficiency of the motor is taken into account. If, for instance, the efficiency at $300 \mathrm{rev}$. shown in Mr. Slichter's Fig. 4 would be made as high as on direct-current motors, then the power-factors with the same magnetizing and other conditions, would have been approximately four per cent 1ower. This lower power-factor would not have made any harder condition on the supply circuit, but actually would have made a somewhat easier condition, as the supply system would have furnished about eight per cent less kilovoltamperes. For lower speeds this difference in power-factor will be greater, and less for higher speeds. A high power-factor at start, obtained by the use of resistance in series with the motor by high internal losses which do not represent torque, is therefore a detrimental condition rather than a good one, as it means increased kilovolt-ampere expenditure for a given torque. This is merely given as an illustration showing that power-factor in itself is not a true indication of conditions, but must be accompanied by other data; this is not a criticism of these motors, $b u^{\imath}$ is a general condition, found to a greater or less extent in all alternating-current motors, 
In conclusion the speaker wishes to ask a few questions in regard to some of the points brought out in these papers. One point in connection with Fig. 1, Mr. Slichter's paper, is the high power-factor of 50 per cent shown at start. The sneaker would like to know how this power-factor can be so high when the motor is doing no work at this point. Is this power-factor due to high internal losses of the motor, or is an external resistance added? The speaker has tested a number of series and repulsion motors, and for motors of this capacity, as a rule, the-powerfactors have been somewhat lower than this; the losses in the repulsion motor for a given starting torque are always greater than in the straight-series motor, but the volt-ampere input for the same torque was also greater so that the power-factors in some cases were very nearly equal.

Also, Mr. Slichter states that the motor shown in Fig. 4 is for constant-speed running; does this mean running at a practically steady load, or is this motor intended for constant-speed work, in the sense of a shunt-wound direct-current motor?

In the comparison with the direct-current motor it is stated that the alternating-current motor remains on the controller for 16 seconds, and the direct-current motor for 25 . Would it not be true that the direct-current motor could remain on the controller for a shorter time, if designed with an unsaturated field, as the alternating-current motor? Is not this a property of an unsaturated motor, rather than an alternating-current motor?

A.S. McAllister:-In the papers presented this evening, mention is made of the fact that the repulsion motor, when driven against its torque, acts as a generator; and when the negative speed becomes of a value such that its product with the torque exceeds the internal losses of the motor, the machine will feed energy back to the line. The analytical equations given in Mr. Steinmetz's paper furnish an explanation of the cause for this action on the part of the machine. These equations may be represented graphically by a simple diagram, and it is believed that the diagram affords a readier interpretation than do the equations.

When the rotor of the repulsion motor is stationary, measuring in truments placed in the primary circuit will indicate an apparent impedance, having power and reaction components. The power component, which will here be termed " resistance," represents the resistance effect of the primary coil, the equivalent resistance effect of the secondary circuit, and the effect of the core loss at starting. In the impedance electromotive force for one ampere-diagram of Fig. 1 - this resistance, $R$, is represented by the line $A B$, while the line $B C$ shows the apparent reactance, $X$, and the cosine of the angle $C A B$ gives the power-factor of the motor at starting. When the motor operates at a speed, $S$, the power component of the apparent motor impedance-volts for one ampere-is increased by a certain value $P S, C D$ in the diagram, $P$ being a coefficient depending upon the relative 
strength of the field which causes the generation of electromotive force in the armature conductors at speed $S$, and hence depends upon the position of the brushes. If the apparent reactance of the coils remained of value $X$ at all speeds, then at speed $S$ the apparent impedance of the motor becomes :

$$
Z_{\mathrm{s}}=\sqrt{(R+P S)^{2}+X^{2}}
$$

and for impressed electromotive force of $E$ the current will be

$$
I=\frac{E}{\sqrt{ }(R+P S)^{2}+X^{2}}
$$

and the power-factor, $\cos 0$, is

$$
\cos D A B=\frac{R+P S}{\sqrt{(R+P S)^{2}+X^{2}}}
$$

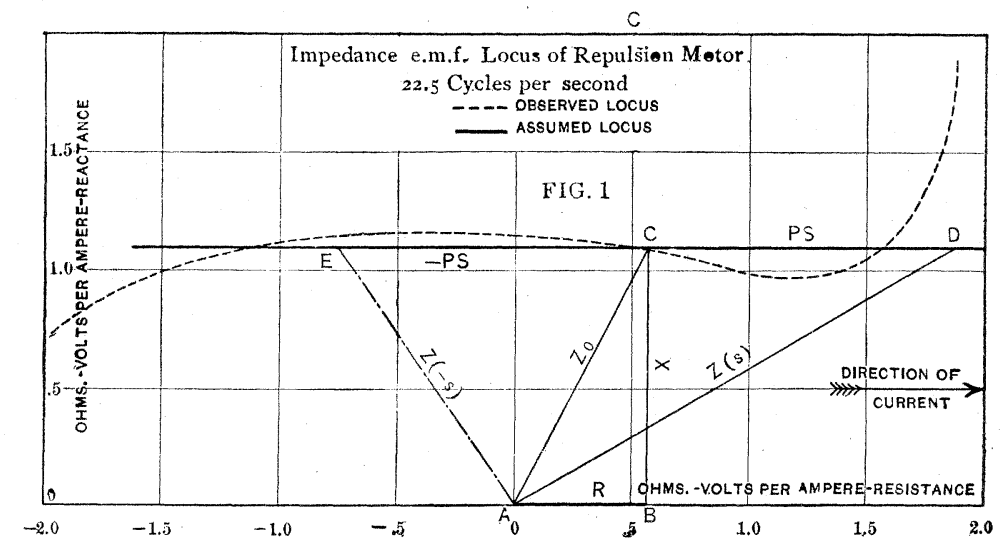

The power input is

$$
E I \cos \theta=\frac{E^{2}(R+P S)}{(R+P S)^{2}+X^{2}}
$$

If, independent of the change in speed of the rotor and variation of the relative values of the primary and secondary currents, the total internal losses of the motor be considered proportional to the square of the primary current, the output would be

$$
I E \cos \theta-I^{2} R=\frac{E^{2} P S}{(R+P S)^{2}+X^{2}}
$$

and the ratio of output to speed, the torque will be

$$
I^{2} P
$$


The discrepancies between the assumptions made and the physical facts involved will be evident from a study of the formation of the above equations. It will be observed that the equations here given will become identical with the approximate equations of Mr. Steinmetz if for each value used above there be substituted its equivalent as given below,

$$
\begin{aligned}
& R=r^{1} \\
& X=X \sin ^{2} \omega+X^{1} \\
& P=X \sin \omega \cos \omega \\
& S=a
\end{aligned}
$$

An inspection of Fig. 1, or of the equations above, will reveal the fact that when $S$ is negative and of a value such that $-P S=$

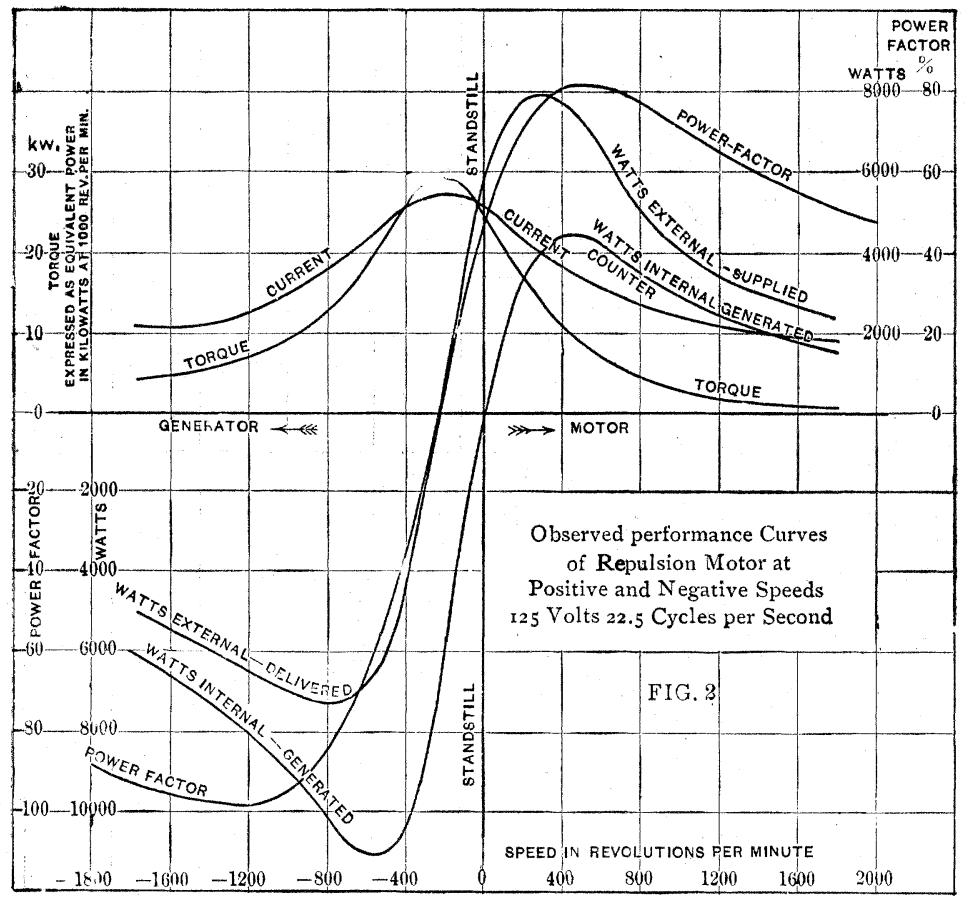

$R$, the apparent impedance of the motor becomes $X$; the current is $E / X$ and represents no energy flowing either to or from the motor; that is, the machine is operating as a generator and supplying its own losses. A further increase of negative speed causes the power component of the apparent motor impedance to take on a negative value; the power-factor becomes negative indicating that energy is flowing from the machine.

The curves of Fig. 2 show the results of tests of a certain repulsion motor while those of Fig. 3 give the calculated performance of the same motor, proper substitution having been made in the equations above. 
In the discussion of the theory of the repulsion motor, $\mathrm{Mr}$. Steinmetz states that the effect of the short-circuit by the brush of active coils tends to a reduction of the power-factor at high speeds; which statement may be taken as an explanation of the fact that the power-factor observed in case of high speeds is much below that calculated, as will be seen from a comparison of Figs. 2 and 3.

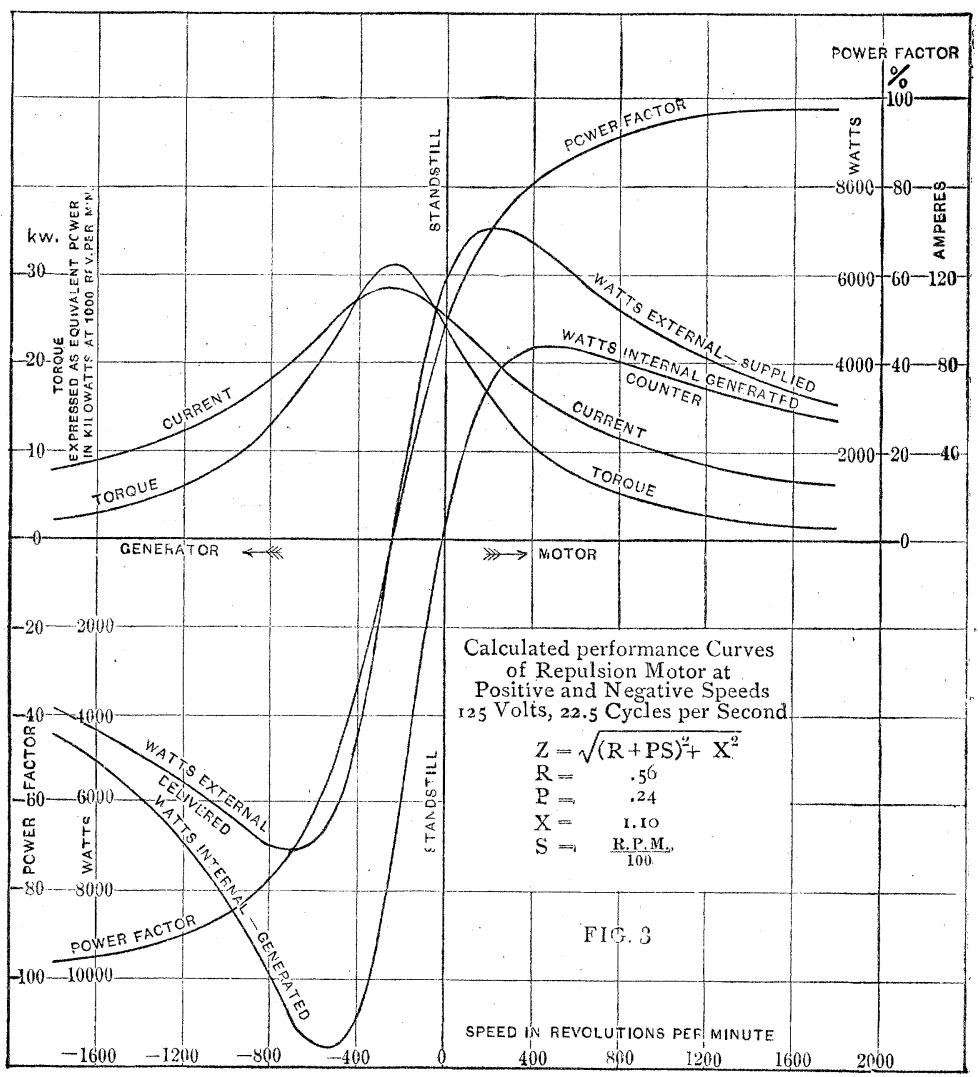

The neglect of the counter-torque, due to the local current flowing in the coils under the brushes, leads also to error in assuming that the repulsion motor tends to increase indefinitely the speed of its rotor as the applied electromotive force is increased or the load decreased, since in reality experiments show that the rotor tends to reach a certain speed above which, if it be driven mechanically, it acts as a brake.

President Arnold:- Since this discussion may properly be divided and naturally divides itself into two parts, such as motor designing and the application of single-phase alternating-current 
motors to railway work, it would be proper at the present time to give Mr. Steinmetz an opportunity to answer Mr. Lamme's questions and any of the other questions which have been asked.

C. P. Steinmetz:-An alternating-current series motor, to have a reasonably good power-factor, must have a high ratio of armature magnetomotive force to field magnetomotive force; this conclusion, reached ten years ago by Mr. Eickemeyer and the speaker, is agreed to by Mr. Lamme; but there is a limit to the indefinite increase of this ratio, for the reason that the armature circuit is not really non-inductive, and there comes a point when the increase in the armature self-inductance is greater than the decrease in the field self-inductance. For the motor on which experiments were made ten years ago, this ratio was between 3.5 and 4 , but very high frequencies were then used; with lower frequencies the ratio can be increased, yet there is still a limit to its indefinite increase. The fact of self-inductance in the armature explains the reason that the power-factor, calculated as Mr. Lamme calculates it-on the assumption of a noninductive armature-gives higher values than tests show. Moreover, a brush shift of $16^{\circ}$ is not equivalent to a ratio of 1 to 5 . The two sections of the winding must be combined approximately vectorially, giving a ratio of sine $16^{\circ}$ to sine $74^{\circ}$, or about 1 to 2.86 , and this ratio will correspond to a lower power-factor than that obtained for a ratio of one to five.

One great advantage of the modern repulsion motor is that it uses a pole-arc of $180^{\circ}$, or the largest section of air-gap possible, and so secures at low speeds so high a power-factor that it is not necessary to go to speeds far above synchronism. The result thereof is a small number of poles, great number of commutator segments per pole, hence small short-circuit current in starting and running, and a low-speed commutator.

The repulsion motor has inherently such a high power-factor that a large air-gap and a high flux-density may be used, hence with heavy currents magnetic saturation is approached or passed in part of the magnetic structure, and a bend occurs in the torque curve. It is not feasible to use flux-densities quite as great as in the direct-current series motor. Further, at low speeds a point must be reached where the electromotive force of rotation becomes small compared with the electromotive force of self-induction, however small the latter may be, and below this speed the torque and current do not increase, but remain constant both in the repulsion and in the series motor.

There is no doubt that a motor designed as a series motor will give larger output and greater efficiency than the same motor used as a repulsion motor, as Mr. Lamme states; but, on the other hand, a motor designed to be repulsion motor and then run as a series motor will give the opposite result - that is, the output will be greater and the efficiency better, when operated as a repulsion motor. The reason for this is simple, and is found in the fact that a series motor must have definite polar projections, 
whereas the repulsion motor uses a distributed winding, similar to that of an ordinary induction motor; hence, a low inductance is obtained with a correspondingly high power-factor. Thus the transformer feature of the repulsion motor requires a change in design, and makes a comparison of the relative performance of a particular design first, as a repulsion, and then as a series motor, meaningless.

P. M. LinCOLN :-The observations made on a car of about 40 tons, equipped with four 125-hp. alternating-current series motors, show that when in motion at speeds not above four or five miles an hour, the motor sends power back into the line on reversal as indicated by a wattmeter; the wattmeter reverses when the fields are reversed and current is on. The same characteris $t$ ic has also been reproduced in shop tests, where a straightseries motor has been used as a generator to feed power back into the shop circuit to which it is connected, by driving it suitably by another motor. Another characteristic of the alternating-current series motor is that it will always build up as a direct-current generator when the reverse switch is thrown and no power applied; it acts just the same as the direct-current series motors and can be used in the well-known way as an emergency brake. In fact on one occasion, when the air brakes failed, when the car was running at high speed, this emergency brake was thrown and the car stopped very promptly, just as it would with a direct-current motor.

On page 6, referring to the speed-time curves, Mr. Slichter gives the comparative inputs for alternating and direct current, for the same car running the same distance, as 0.72 kilowatthours for the direct and 0.685 for the alternating current, approximately for the alternating current five per cent less energy; but the rheostatic loss for the direct-current curve amounts to approximately 15 per cent of the total curve area. Heaccounts for the difference of five per cent, whereas the curve areas seem to indicate that there should be a difference of at least 15 per cent, since the alternating-current motor does not include the rheostatic losses.

Mr. Slichter:- The fact that the power-factor at starting, shown in the first curve, is as high as 50 per cent, is due to the large losses and low efficiency of this particular motor. This motor was designed some time ago, when the matter of commutation was considered to be very serious, and radical precautions were taken to prevent bad sparking at the expense of efficiency. Recent experience has shown that these precautions are not necessary and as a result it has been possible to improve the efficiency.

This low efficiency, 80 per cent, is also the cause of the small difference between the energy consumption of the alternatingcurrent motor and the direct-current motor as the length of run increases. The low efficiency of this motor at high speeds counterbalances the saving effected by potential control during 
acceleration. With a later design of alternating-current motor this efficiency would be much higher and the net saving by the use of potential control would be much more noticeable.

Referring to the motor of Fig. 4, it is intended for hauling heavy trains at constant slow. speeds with few stops; in other words, long-distance freight haulage.

The fact that the controller of the alternating-current motor is cut out in so short a time, 16 seconds as against 25 for the directcurrent motor, is due to the steep speed-characteristic of the motor.

In reply to Mr. Lamme's criticism of the curves, they are intended to give a general view of the characteristics of the repulsion motor and are for this purpose extended over values of current outside the working range of the motor? In this range of the curves there are several points of curvature which are difficult to check exactly, since only a few points were plotted to show the general direction.

RALPH D. MERSHON:-Mr. Slichter speaks of voltage control; what method of voltage control was used on these motors, was it such as would be used commercially? What were the comparative weights of alternating-current motors, and directcurrent motors, for the same work and for approximately the same performance, either on the basis of efficiency of the motor itself or the total efficiency of acceleration? What were the relative weights of the motor and of the complete car equipment, for alternating current and direct current? Also what pressure would he use with the alternating-current motor? $\mathrm{Mr}$. Slichter says these motors can be wound for high-pressure; how high? and what method of control would be used in that case? Another important point is, can the repulsion motor be so designed as to be a good repulsion motor, and yet be a pretty good direct-current motor?

It will be quite difficult to get the people responsible for existing large direct-current railway systems up to the point of changing over any considerable portion of the road at one time. The change can be much more easily accomplished if it can be made more or less gradually as, for instance, by changing outlying sections first, and this can be done if the motor is such as can be used on both the direct-currentlines and alternating-current lines. As a matter of interest, what was the number of poles of the motors represented by these curves?

A. H. Armstrong:- The curves given on the first three pages of Mr. Slichter's paper refer to four-pole motors and the curves in Fig. 4 to a six-pole motor, all operating at 25 cycles. Mr. Mershon stated the matter about right when he asked if a repulsion motor could be designed to operate well on alternating current and at the same time be operative on direct current. It is not possible to get equally good results with both alternating current and direct current, but a good alternating-current motor will give sufficiently good results when operated on direct current to enable a car so equipped to operate over direct-current lines. 
The repulsion motor can be wound for 2000 or 3000 volts direct, without the use of transformers; but experience in operating with such pressures on railway equipments is yet to be had, and it is not known how much deterioration will be produced by the constant jarring and crystallizing of the insulation. Should it become too expensive to maintain high-pressure motor winding, it will be necessary to resort to the use of transformers; with the high-pressure motors, control will be effected by either a compensator or by the insertion of resistance, as in the present direct-current control.

If a car stops once in five miles, the small difference in efficiency in starting between rheostatic and pressure controlwould have practically no effect upon the general efficiency of the system as a whole. A gain of one or two percent in the average car energy would not compensate for the additional complication. It will be entirely feasible to consider 2000 or 3000 volts on the field of the repulsion motor without any intervening apparatus, but this, as stated before, will be largely determined by commercial operation. The repulsion motor having no metallic connection between field and armature, and thus not being in any way limited by commutation can have its field wound for as. high-pressure as operating conditions will permit.

No definite figures can be given for the relative weight of alternating-current and direct-current railway motors. The capacity of direct-current motors has been very carefully worked out during the past few years; but the alternating-current motor is not in commercial operation, it has not been possible to obtain experimental data upon its relative capacity and hence its weight and size compared with the direct-current motor must remain an open question for the present. The alternating-current motor will undoubtedly weigh somewhat more than the direct-current motor for the same service performed, but the percentage increase cannot be stated with any degree of accuracy at the present time.

In a high-pressure motor system using trolley pressure directly on the field, the control system should not weigh much more than that required for direct-current motors. If, however, a lowpressure motor is used with the high-pressure trolley, it will require the installation of a step-down transformer, which weight. must be considered in addition to the regular control system. The average energy consumption of some of our suburban cars. reaches as high as 75 to $100 \mathrm{kw}$., and the addition of a $100-\mathrm{kw}$. transformer of either the oil-cooled or the air-blast type will. constitute an appreciable addition to the weight of the car equipment.

President Arnold:- The question of the destruction of the insulation or crystallization of the metal used in the construction. of high-pressure motors has been pretty thoroughly answered in. practice by the use of such high-pressure motors abroad; the line in Italy, using 3000-volt motors, has been operated for two 
or three years and no such objection has been used against it. If abroad they can build motors which can run successfully under that pressure, we should be able to do the same.

C. P. Steinmetz:-The present alternating-current motor, either series or repulsion type, has a somewhat lower efficiency and is somewhat heavier than the direct-current series motor, but this difference is less than would be expected; the principles involved in the design of the two types are quite different, so that no direct comparison of one with the other can be made.

There is no inherent reason why the efficiency and weight of the alternating-current motor should not be on a par with those of the direct-current motor, after the type has been further developed.

RoBERT LUNDELL:-A possible reason why the efficiency of the alternating-current motor is not as great as that of a directcurrent motor is because the hysteresis losses and the eddy currents are bound to be larger in the alternating-current motor. In the alternating-current motor we have to take care of the eddy currents and hysteresis losses in an apparatus which must weigh more much than the armature of the direct-current motor. As the hysteresis or core losses are due to the cycles per second, the density of the magnetism and also to the weight, it seems to me that the weight is bound to be greater in the surrounding frame, that is, the field magnets which surround the armature of the alternating-current motor. Is not that one reason why the efficiency of the alternating-current motor can not possibly be as high as that of the direct-current motor?

O. S. LYFORD, JR.-In a direct-current motor, we can get 200 horse power in a motor on the truck, or 400 horse power in a pair of wheels on one truck; will Mr. Slichter say whether the 175 horse power motor described on page 66 is of such dimensions that it could be placed on the ordinary interurban car-truck with 33 or 36 inch wheels? How large an alternating-current motor can we get for a 33 or a 36 inch wheel? In other words, what is our limit at the present time in the motor-car field?

W. I. SLICHTER:-That motor is designed for locomotive work and would not be of dimensions to go under a car having 33 inch wheels.

The ideas on this subject are developing very rapidly; nne could not say that a 100 horse power motor could be placed there, but perhaps it would not be advisable to say that a larger motor could not be.

B. G. LAMME:-The Westinghouse Electric and Manufacturing Company is building 150 -hp. motors which will go in on 33-inch wheels. closely; easily on 36-inch wheels It has been determined that we can put 200 -hp. motors on 36 -inch wheels. That is one limit.

PRESIDENT ARNOLD:- If you gentlemen who build railroads will call for the motors, you will probably get them.

H. A. WAGNER:-Does not the repulsion type of motor take 
somewhat more copper in the field than the series motor? and in reversing the former, is it proposed to move the brushes or to feed the current into the stator at a different point?

C. P. Steinmetz:- The repulsion motor can be reversed by changing the point of entrance of the current to the primary wires, without changing the brushes; thus the primary winding may have current brought to it at several different points in the repulsion motor, and the brushes need not be shifted. The relative amounts of copper in the armature and field may be varied to some extent to suit the design, but there is no essential difference in the proportions of material.

[Communicated after Adjournment by B. G. Lamme.]

In the discussion of the papers of Messrs. Steinmetz and Slichter, the writer indicated in a very general way some of the reasons which led him to the advocacy of the straight-series motor. Some of the more important of these reasons can be classified as follows:

Lighter weight with a given output and given performance.

Larger air-gap with a given input at start.

Less short circuiting at commutator at start, with a given starting torque.

Ability to operate the motor on various pressures by simply using taps on the transformer which supplies the motors. For instance: a motor can be run at 3000 volts on one part of the line, or at 500 volts on another part by simply feeding the trolley circuit into a different point in the transformer. Also the transformer can be readily stepped-up to a slightly higher pressure, thus allowing a higher normal speed and ability to make up for delays. This general arrangement will be of especial importance in heavy railway work, now represented by steam service.

The motor will be normally wound for low pressures. It has been claimed that the advantage of thealternating-current motor for railway work is that it can be wound for high-pressure, but, on the other hand, it enables us to wind the motor for lowpressure. Taking into account the troubles that are found with direct-current motors at 500 and 600 volts, the writer believes that one of the greatest advantages of the alternating-current motor is that the motor itself can be wound, if desired, for much lower than 500 volts, while a pressure of 2000 or 3000 can be supplied from the trolley. The writer does not believe that a pressure of 3000 should be supplied directly to the motor. This judgment is based partly on European experience with high pressures on railway motors. A motor wound for this pressure will necessarily be heavier than one wound for low-pressure, even if the same type of motor is retained. Therefore, if low-pressure is to be used on the motor with a step-down transformer on the car, then the writer considers that the repulsion type of motor, being naturally heavier than the straight-series, is used at a great disadvantage, as the equipment contains not only transformers in the motors themselves, but also an external transformer. 
A further condition, applying to all alternating-current motors, is the fact, already published, that there is an active pressure between turns on the field of motors. This is of considerable importance considering the overload capacity of such motors. In the direct-current motor a short circuit can occur between two or more field-turns without immediately disabling the motor. In the alternating-current motor a similar short circuit will disable the motor. Therefore if the alternating-current motor is to meet the same conditions of service as the direct-current motor, it will require much more insulation in its windings than that required in the direct-current motor, and this in turn means that the motor must be either increased in size, or must be wound for lower pressure. The writer therefore believes that whether straight-series or repulsion motors are used, the motor should be wound for a relatively low pressure.

Mr. Steinmetz's suggestion that these conclusions in regard to relative weights of straight-series and repulsion motors were based on the use of series motors operated as repulsion motors, just as his conclusions were based on repulsion motors operated as series motors, is incorrect; for these conclusions have been drawn from designs of straight-series and of repulsion motors, each type being proportioned for its own special method. of operation; the writer has designed a number of motors of each type, and a repulsion motor of 125 horse power capacity was constructed for the purpose of investigation.

Mr. Slichter states that the discrepancies in the curves of Figs. 1 and 4 , are due probably to the complicated nature of the calculations of these curves; but his paper states that the curves of Fig. No. 1 " are taken from tests on a 60 -hp. 25 -cycle, 500-volt motor." If these curves were obtained entirely by calculation, there could be wide discrepancies which might have been overlooked, if the final results were not checked with each other.

Mr. Steinmetz states that one advantage of the repulsion motor over the series motor lies in the fact that the magnetic leakage should be less, due to the distributed windings of the primary; but he overlooks the fact that in a series motor designed for high power-factor, the magnetic leakage of the exciting field-winding can be made extremely low; the leakage of the two motors will be practically equal if the primary fields are wound for equal pressure; if, however, the repulsion motor field is wound for highpressure, with a comparatively small number of slots, the magnetic leakage will be higher than on the low-pressure motor, and will there ore be higher than on the series motor. Mr. Steinmetz's opinion of straight-series motors may be based on data obtained about ten years ago. There has been considerable improvement in the art since then, and the development of this motor for commercial railway service has undoubtedly led to proportions and forms of construction not contemplated ten years ago. 


\section{[Communicated after Adjournment by Chas. F. Scott.]}

The papers and their discussion are notable on account of several omissions. The subject under consideration, namely, the single-phase railway motor, brought forth no question as to the practicability of the single-phase system; it elicited no mention of the polyphase railway motor; it led to no questions regarding the matter of commutation of alternating currents.

It must be borne in mind that it is less than a year and a half since the first authoritative announcement of the single-phase railway motor was made. 'The announcement was the occasion of much surprise and many were sceptical as to the question whether the single-phase motor was practicable or even possible. The practicability of the single-phase motor is now received without question, and it is tacitly accepted that the single-phase motor and the single-phase system are factors of very serious consequence in the immediate extensions of railway work.

At the Buffalo Convention of the Institute in 1901, a paper was read by $\mathrm{Mr}$. Berg in the discussion of which Mr. Steinmetz and the writer took part. We concurred with the conclusions of the paper that the polyphase motor was not suited for railway work, and that the efforts of European engineers to introduce polyphase motors for railway work were not along lines which would prove a general solution of the railway problem. There were criticisms at that time and subsequently in the technical press setting forth the backwardness of American engineers and indicating the forward steps which were being made in Europe in the introduction of alternating current for railway work. Our contention, however, was not against the alternating current but against the inauction motor for railway work. It is notable that since it has been stated that there is an American single-phase motor, European engineers have taken up the development of single-phase apparatus, indicating thereby the shortcomings of the polyphase railway motor. It is significant that the polyphase motor did not come up for consideration in the general discussion of the single-phase motor in connection with the Steinmetz and Slichter papers.

There was some disappointment in connection with a meeting of the Institute a little over a year ago at which the single-phase series motor was presented, because certain specific data regarding the design of the motor, particularly in connection with its commutation, were not given in connection with the general engineering presentation of the single-phase system. There seemed to be an idea that there was a mysterious something which had been introduced into the motor as a panacea for the evils of alternating-current sparking, which was being withheld. The papers now under discussion do not deal with the particulars of commutation. The general statement that the single-phase motor, whether of the repulsion type or of the straight-series type, does commutate and commutate successfully, was accepted without question and without awaking any apparent curiosity 
as to the methods or devices by which it is accomplished. This illustrates how quickly a new thing passes from the category of that which is doubtful and incredible to that in which it is an accepted fact, from which important commercial results are expected to follow as a matter of course.

The present papers and their discussion deal almost entirely with the presentation of one type of single-phase motor and a comparison bet veen it and a second type. The motor, however, is but one feature, although the fundamental one, $c f$ the singlephase system. It is the general system which the motor makes possible, as was intimated in the remarks of President Arnold, which is the thing of real consequence. The significant result of the introduction of this motor is not the motor itself, but it is the use of alternating current throughout the whole system; it is the omission of synchonous converter sub-stations with synchronously running apparatus, rendering the present distribution system one which is inefficient, cumbersome, and sensitive in operation; it is the use of high-pressure and the resulting economy in transmission; it is the many advantages found in the construction of controllers for heavy alternating current over those for heavy direct current; it is the advantages which come from pressure control instead of resistance control; it is the readiness with which energy may be returned to the circuit by the alternating-current motor having pressure control (which is not practicable with the direct-current motor having pressure resistance control); it is the lessened concentration of energy in alternatingcurrent circuits in case of short circuit; it is the safety to the car equipment on account of the non-flashing of alternating-current motors, thus avoiding one of the serious difficulties in directcurrent motors on large equipments; it is the freedom from electrolysis, and, in short, it is the superiority shown by the alternating current over the direct current in practically every element from generator shaft to motor shaft, by which the limitations found in the direct-current system are removed and a new field, the bounds of which are not yet determined, is opened in the development of electric railway service. The outcome of the synchronous converter which has been so great a factor in the railway extensions of the past half dozen years has been the suburban and interurban railway. The single-phase motor opens the way to a new system which has in it elements which promise far greater developments than those which followed the introduction of the synchronous converter.

\section{[Communicated after Adjournment by B. A. Behrend.]}

The writer wishes again to illustrate by a few diagrams the close resemblance between the alternating-current series motor with a compensating coil for the cross-ampere turns of the armature on the one hand and the repulsion motor on the other. Fig. 1 shows the straight-series motor; Fig. 2 shows the series motor with compensating-coil. In order to obtain a high power- 
factor it is necessary, as has also been pointed out by Mr. Steinmetz and Mr. Lamme, to make the armature magnetomotive force considerably greater than the field. This is indicated in Fig. 2 by showing the field-coil $F$ with few turns and the compensating-coil $C$ with a large number of turns; instead of passing

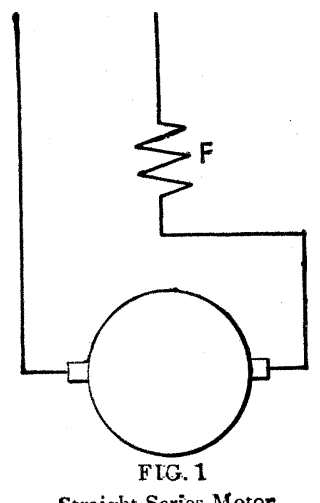

Straight Series Motor

the field current through the compensating-coil $C$, this coil can be short-circuited itself as illustrated in Fig. 3. The main current also can be passed through the compensating-coil $C$ in series with the field $F$ and the armature be short-circuited on itself, thus making the armature the secondary and the compensating-coil

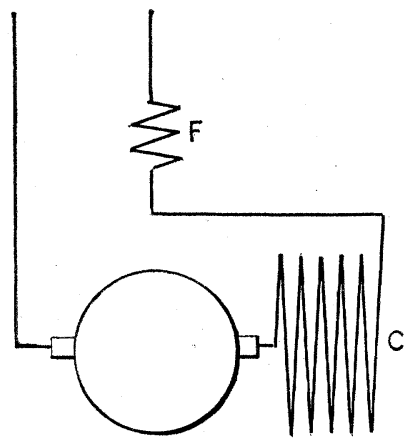

FIG. 2

Series Motor with Compensating

Coil

the primary instead of the reverse, as in Fig. 3. The coils $F$ and $C$ may be replaced by one resultant-coil $R$, leading to the repulsion motor Fig. 5 .

From the above considerations, it follows that, with very close approximation, the torque, power-factor, and the 
efficiency of the compensated series motor represented in Figs. 3 and 4 are equal to those of the repulsion motor Fig. 5 . There is no reason why the power-factor of the repulsion motor should be better than the power-factor of the compensated series motor

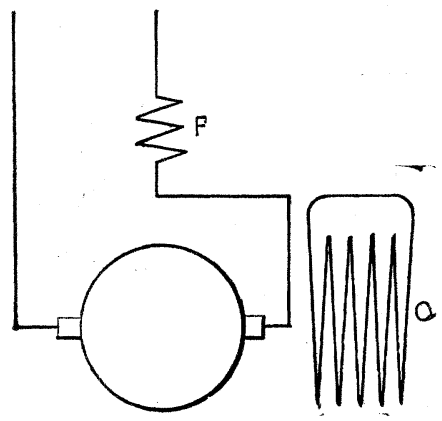

FIG. 3

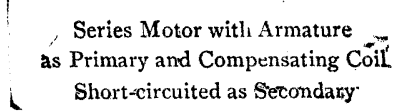

of Fig. 2; and Mr. Steinmetz's Fig. 4 showing the comparison between a repulsion motor and an Eickemeyer compensated series motor is therefore most misleading. If the motors are

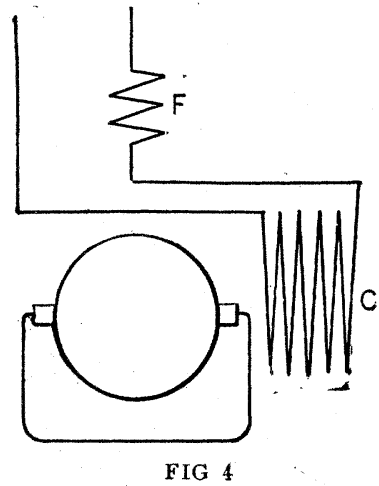

Series Motor vith

Compensating Coil as Primary, and Armature Short-circuited as Secondary (Repulsion Motor)

designed with the same degree of care they should have approximately equal power-factors at all speeds. Mr. Steinmetz says: "The secondary current of the transformers, however, lags slightly less than $180^{\circ}$ behind the primary current; that is, con- 
sidering it in the reversed direction, is a leading current with regard to the primary current. The current in the armature in Fig. 3 is therefore a leading current with respect to the line current and so not only does not add an additional lag, but reduces the lag caused by the self-induction of the field-exciting coil."

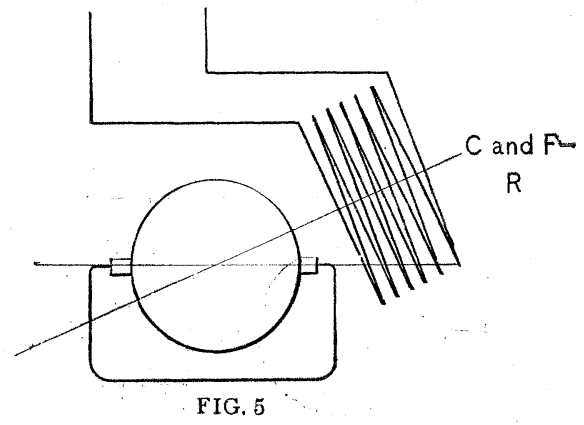

Repuision Motor Field and Compensating

Coil Combined

Mr. Lamme says on this point:

"In either the series or repulsion type of motors, high power-factors, especially at low speeds, are directly dependent upon this fact of high ratio of armature to field, and with a high ratio, high power-factors should be obtained without crediting the result to leading currents in the

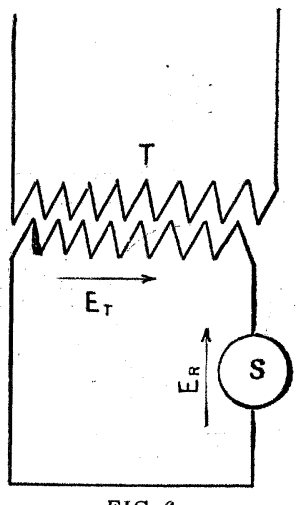

FIG. 6

$\mathbf{E}_{\mathrm{T}}$ represents e.m.f. of transformation

$\mathrm{E}_{\mathrm{R}}$ " " " rotation

\section{armature."}

The fact is, however, that the secondary current in the repulsion motor, beyond a certain speed of the armature, leads in respect to the primary electromotive force as in a transformer in which the secondary is working on self-induction and 
capacity, or on self-induction and an over-excited synchronous motor. The primary current, however, is always a lagging current in regard to its impressed electromotive force. The repulsion motor is like a combination of a transformer with a synchronous motor, the electromotive force of which is coincident in

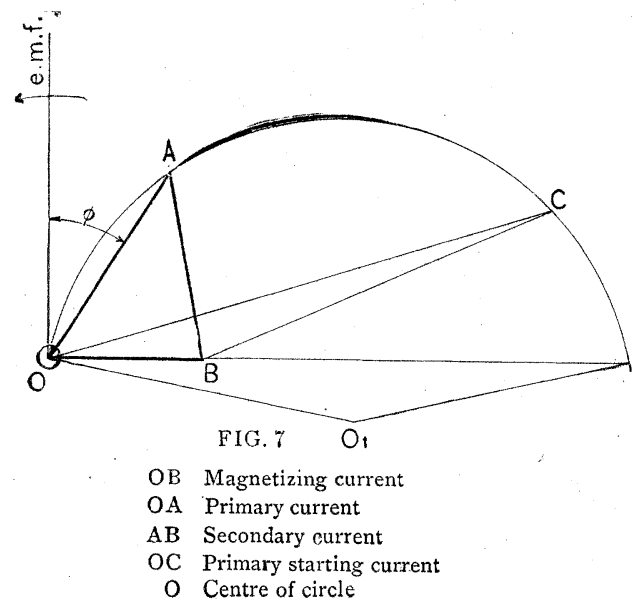

phase with the primary current (Fig. 6). The electromotive force of transformation $E_{T}$ and the electromotive force of rotation, $E_{R}$ (the latter is proportional to the speed of rotation) are in opposition and give a resultant electromotive force, which

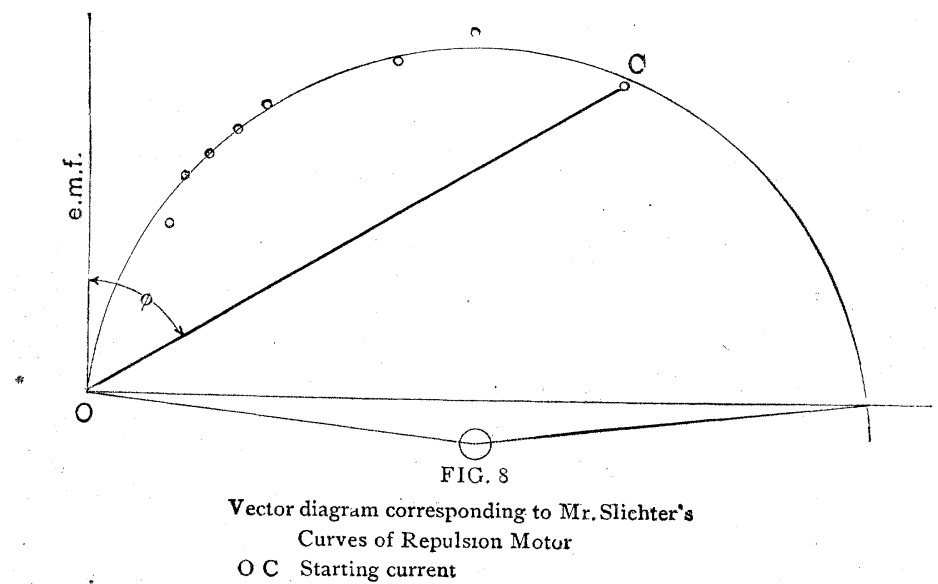

produces the current through the ohmic resistance of the shortcircuited armature. With Fig. 6 in mind it is easy to see that the secondary current leads the primary electromotive force at certain speeds of the armature. The polar diagram brings out 
this point even more clearly. Fig. 7 represents the vectordiagram of the repulsion motor with a small resistance in the armature. This is the theoretical vector-diagram of the repulsion motor as developed a few months ago by Messrs. Osnos and Blondel; the writer's tests, as well as Mr. Slichter's curve, agree closely with this diagram; the polar diagram corresponding to Mr. Slichter's curves is represented in. Fig. 8.

As has also been pointed out by Messrs. Osnos and Blondel, the torque of the repulsion motor, in contradistinction to the series motor, vanishes at a finite speed, hence the repulsion motor is not so likely to run away as the series motor. For the sake

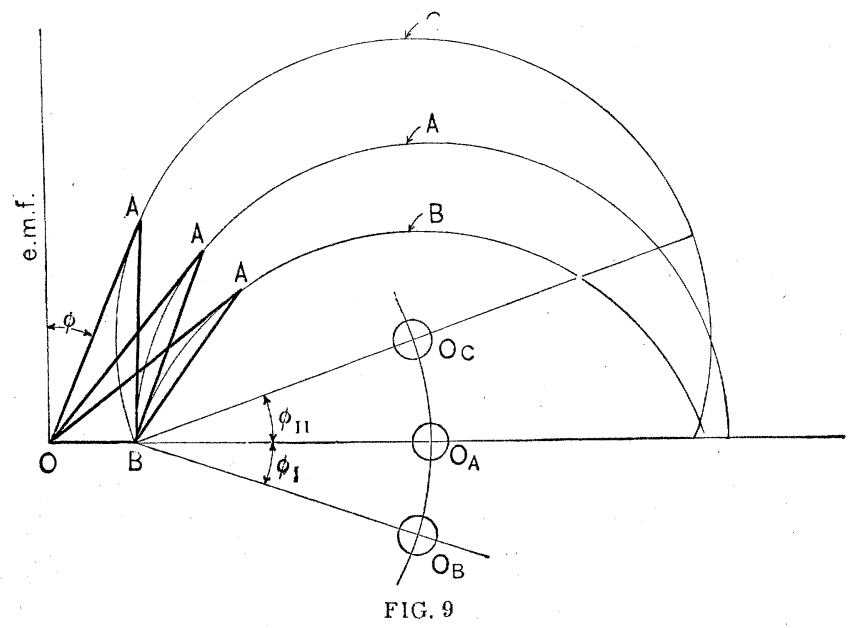

O B Primary magnetizing current

O A Primary current

A represents locus of vector of primary current with resistance in

$\left.\begin{array}{lllllll}\text { B } & \text { C } & \text { " } & \text { " } & \text { " } & \text { " } & \text { " inductance in }\end{array}\right\}$ secondary

of comparison, Fig. 9 shows the vector diagram for the polyphase induction motor with $(A)$ resistance in the secondary, $(B)$ reactance in the secondary, $(C)$ capacity in the secondary. (See Chapter VIII. of the writer's book "The Induction Motor." from which these diagrams are taken.) It is clear that the compensated series motor represented in Fig. 2 can be made with a better power-factor than the compensated series motors represented in Figs. 3 and 4, and hence the compensated series motor in which the main current passes through the compensating-coil, everything else being equal, has a higher power-factor than the repulsion motor, instead of a lower powerfactor, as Mr. Steinmetz seems to think.

Mr. Lamme's remarks are most interesting and instructive, but the writer believes Mr. Lamme is not correct in stating that the 
power-factor of the repulsion motor is lower than that of the series motor, a conclusion which, by the way, is diametrically opposed to that at which Mr. Steinmetz arrived. Mr. Lamme assumes that the inductance of the armature is negligible, and then draws a parallel between the straight-series motor and the repulsion motor as illustrated by Fig. 4 in this communication. Granting the assumption of a non-inductive armature (which is by no means permissible), Mr. Lamme's conclusions are right, but on that assumption the compensated-series motor would give no better power-factor than the straight-series motor, which is obviously untrue. Mr. Lamme's reasoning and his conclusions have therefore to be modified, and instead of stating as he does that the power-factor of the repulsion motor is lower than the power-factor of the series motor, it should be stated that the power-factor of the repulsion motor is slightly lower than that of the compensated-series motor, but considerably higher than that of the straight-series motor. From the English patent specification, it appears that Mr. Lamme's motor is a compensated-series motor, corresponding to Fig. 3 in this communication.

In order to obtain high power-factors, it is necessary to design both the series motor and the repulsion motor on the same principles that have been found of importance in the design of induction motors. The successful series motor, however, has to be built with defined poles. Very good repulsion motors can be built for 60 cycles, and maximum power-factors of 90 to 95 per cent can be obtained even on small machines for comparatively low speeds. Such motors have very excellent series characteristics and can be used wherever the direct-current series motor is satisfactory.

By W.S. Franklin.-(Communicated after Adjournmont):On May 19, 1903, the writer had the privilege of debating some points in alternating-current theory with Mr. Steinmetz before the Schenectady Branch of the Institute. During the visit which preceded this debate, the writer discussed the theory of the repulsion motor, at length, with $\mathrm{Mr}$. Steinmetz. The writer had very recently worked out the theory of the repulsion motor, outlined below, and his results are in substantial agreement, with Mr. Steinmetz's; but knowing that Mr. Steinmetz had been working at the matter much longer, the writer urged him to present his work: to the InstituTe, at an early date.

At the time of the conference, it was clear to both Mr. Steinmetz and the writer that the ideal theory as given in Mr. Steinmetz's paper, and as outlined below, did not take account of the very considerable distortion of the electromotive force wave due primarily to the fact that the two fluxes $\phi^{\prime}$ and $\Phi^{\prime \prime}$ (see below) are not harmonically distributed around the periphery of the 
machine. A first approximation to a correct theory is based upon the assumption that the fluxes are harmonic both in time and in space. A second approximation would be to consider the departure of the fluxes from harmonic space distribution, basing the calculations of this departure upon the first approximate solution by taking account of the magnetomotive forces generated in the several parts of the windings by the harmonic current values arrived at by the first approximation. Whether a third approximation, based upon calculated departures of the fluxes from harmonic variation in time, would be required for practical purposes, could be perhaps more cheaply answered by making a motor and comparing actual tests with second approximate calculations than by the arithmetical determination of the magnitude of the corrections introduced by the third approximation.

The development of the theory (first approximation) of the repulsion motor following is essentially the same as that of $\mathrm{Mr}$. Steinmetz; it is given here because of the slightly different point of view, and because it is an example of the right-handed notation $(r+j x$ instead of $r-j x$.)

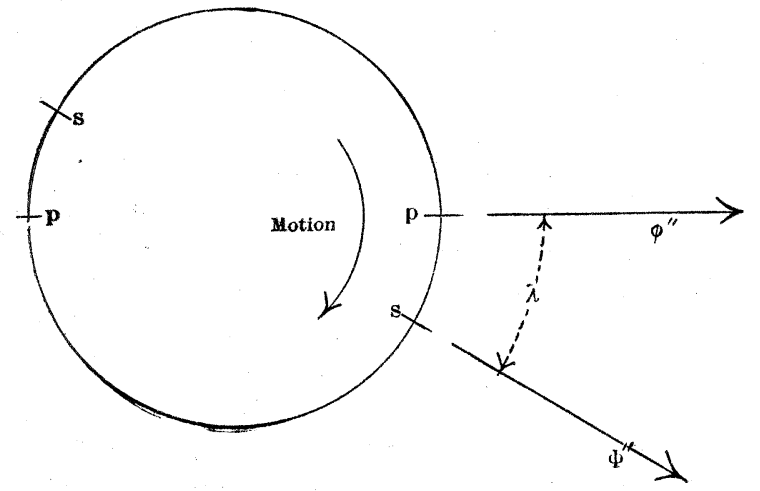

FIG. 1.

2. Outline of Theory of Repulsion Motor.

Fig. 1 shows the axis of commutation $s s$ of the armature (secondary-tap axis), and the corresponding axis, $p p$, in the primary member of a repulsion motor. The primary current produces an alternating flux $\phi^{\prime}$ at full primary frequency $f^{\prime}$ in the direction of the primary-tap axis, and the secondary or armature current produces an alternating flux $\Phi^{\prime \prime}$ at full primary frequency in the direction of the secondary-tap axis.

Let $e^{\prime}$ be the value of and $E^{\prime}$ the complex expression for primary impressed voltage.

$f^{\prime}$ the primary frequency.

$i$ ' the value of the primary current. The primarycurrent vector is taken as the reference axis and therefore $i^{\prime}$ is also the complex expression for the primary current. 
$\phi^{\prime}$ the value of the alternating flux due to the primary current $i^{\prime}$.

$\theta^{\prime}$ the phase-angle between $E^{\prime}$ and $i^{\prime}$, positive when $E^{\prime}$ is ahead of $i^{\prime}$.

Then

$$
\phi^{\prime}=k i^{\prime}
$$

in which $k$ is the flux produced by unit primary current; and

$$
E^{\prime}=e^{\prime}\left(\cos \theta^{\prime}+j \sin \theta^{\prime}\right)
$$

Furthermore, let $Z$ be the number of conductors in the primary winding; the number of secondary conductors being assumed to be the same for the sake of simplicity.

$r_{1}$ the resistance of the primary.

$x_{1}$ the reactance value of magnetic leakage at primary frequency.

$f^{\prime \prime}$ the speed of the armature in cycles per second.

$i^{\prime \prime}$ the value of secondary current and $I^{\prime \prime}$ its complex expression.

$r_{2}$ the secondary resistance.

$\theta^{\prime \prime}$ the phase-angle between $i^{\prime}$ and $i^{\prime \prime}$, positive when $i^{\prime \prime}$ is ahead of $i^{\prime}$.

$\phi^{\prime \prime}$ the value of the alternating flux due to the secondary curThen rent $i^{\prime \prime}$, and $\Phi^{\prime \prime}$ its complex expression.

$$
\Phi^{\prime \prime}=k I^{\prime \prime}
$$

in which $k$ has sensibly the same value as in equation (1); and

$$
I^{\prime \prime}=i^{\prime \prime}\left(\cos \theta^{\prime \prime}+j \sin \theta^{\prime \prime}\right)
$$

To establish the general equations of the repulsion motor we must consider (a) the voltages induced in the primary. Placing the sum of such induced voltages equal to $-E^{\prime}$ gives one equation; and $(b)$ the voltages induced in the secondary. Placing the sum of such induced voltages equal to $+r_{2} I^{\prime \prime}$ gives another equation.

Equation of Primary Voltages.-The total impressed primary voltage is made up of the following parts:

(a) The part $\left(r_{1}+j x_{1}\right) i^{\prime}$ used to overcome primary resistance and magnetic leakage reactance.

(b) The part $+j \cdot \frac{\phi^{\prime} Z f^{\prime}}{\sqrt{2}}$ used to balance the electromotive force induced in the primary by the pulsating flux $\phi^{\prime}$.

(c) The part $+j \cdot \frac{\Phi^{\prime \prime} Z f^{\prime}}{\sqrt{2}} \cdot \cos \lambda$ use to balance the electromotive force induced in the primary by the pulsating flux $\Phi^{\prime \prime}$.

Therefore:

$E^{\prime}=\left(r_{1}+j x_{1}\right) i^{\prime}+j \cdot \frac{\phi^{\prime} Z f^{\prime}}{\sqrt{2}}+j \cdot \frac{\phi^{\prime \prime} Z f^{\prime}}{\sqrt{2}} \cdot \cos \lambda$ 
Equation of Secondary Voltages.-The total electromotive force induced in the secondary is used to overcome secondary resistance; it is therefore equal to $r_{2} I^{\prime \prime}$, and it consists of the following parts:

(a) The part $-j \cdot \frac{\phi^{\prime} Z f^{\prime}}{\sqrt{2}} \cdot \cos \lambda$ produced by the pulsations of the flux $\phi^{\prime}$.

(b) The part $-j \frac{\Phi^{\prime \prime} Z f^{\prime}}{\sqrt{2}}$ produced by the pulsations of the flux $\Phi^{\prime \prime}$.

(c) The part $+\frac{\phi^{\prime} Z f^{\prime \prime}}{\sqrt{2}} \cdot \sin \lambda$ produced by the motion of the secondary relative to the flux $\phi^{\prime}$.

Therefore:

$r_{2} I^{\prime \prime}=\frac{\phi^{\prime} Z f^{\prime \prime}}{\sqrt{2}} \cdot \sin \lambda-j \cdot \frac{\phi^{\prime} Z f^{\prime}}{\sqrt{2}} \cdot \cos \lambda-j \cdot \frac{\Phi^{\prime \prime} Z f^{\prime}}{\sqrt{2}}$

Equations (5) and (6), together with the equations of definition (1), (2), (3), and (4), are the fundamental equations of the repulsion motor. The assumptions upon which these equations rest and the extent to which these assumptions deviate from the conditions which really exist in a repulsion motor are discussed later. Three things only need be said to clear up the derivation of equations (5) and (6); namely, ( $a$ ) only that component of a flux which is parallel to a tap-axis produces electromotive force by pulsation; (b), only that component of a flux which is at right angles to a tap-axis produces electromotive force by motion; and $(c)$, all induced electromotive forces involved in equations (5) and (6) are of the same frequency $f^{\prime}$. This is due to the fact that the electromotive forces induced in the secondary are referred to the stationary tap-axis SS Fig. 1; the electromotive force (or current) in a given moving secondary wire may be thought of as having a frequency $f^{\prime \prime}$, while its amplitude varies at frequency $f^{\prime}$. This is equivalent to the superposition of two genuine alternating electromotive forces of which the frequencies are $f^{\prime}+f^{\prime \prime}$ and $f^{\prime}-f^{\prime \prime}$ respectively.*.

Substituting the values of $\phi^{\prime}, \Phi^{\prime \prime}, E^{\prime}$, and $I^{\prime \prime}$ from equations (1), (2), (3), and (4) in equation (5), we have:

$$
\begin{aligned}
e^{\prime} \cos \theta^{\prime}+j e^{\prime} \sin \theta^{\prime} & =r_{1} i^{\prime}+j x_{1} i^{\prime}+j \cdot \frac{k Z f^{\prime}}{\sqrt{2}} \cdot i^{\prime} \\
+ & j \cdot \frac{k Z f^{\prime}}{\sqrt{2}} \cdot \cos \theta^{\prime \prime} \cos \lambda \cdot i^{\prime \prime}
\end{aligned}
$$

*2 $\left[\cos \omega^{\prime} t\right] \cdot \cos \omega^{\prime \prime} t=\cos \left(\omega^{\prime}+\omega^{\prime \prime}\right) t+\cos \left(\omega^{\prime}-\omega^{\prime \prime}\right) t$. 


$$
-\frac{k Z f^{\prime}}{\sqrt{2}} \cdot \sin \theta^{\prime \prime} \cos \lambda \cdot i^{\prime \prime}
$$

Substituting the values of $\phi^{\prime}, \Phi^{\prime \prime}, E^{\prime}$ and $I^{\prime \prime}$ from equations (1), $(2),(3)$ and (4) in equation (6) and we have:

$$
r_{2} i^{\prime \prime} \cos \theta^{\prime \prime}+j r_{2} i^{\prime \prime} \sin \theta^{\prime \prime}=\frac{k Z f^{\prime \prime}}{\sqrt{2}} \cdot \sin \lambda i^{\prime}
$$

$-j \cdot \frac{k Z f^{\prime}}{\sqrt{2}} \cdot \cos \lambda \cdot i^{\prime}-j \frac{k Z f^{\prime}}{\sqrt{2}} \cdot \cos \theta^{\prime \prime} \cdot i^{\prime \prime}+\frac{k Z f^{\prime}}{\sqrt{2}} \cdot \sin \theta^{\prime \prime} \cdot i^{\prime \prime}$

Let

$$
\begin{aligned}
& \frac{k Z f^{\prime}}{\sqrt{2}}=a \\
& \frac{k Z f^{\prime \prime}}{\sqrt{2}}=b
\end{aligned}
$$

Then, separating real and imaginary parts, equation (7) becomes

$$
\left.\begin{array}{l}
e^{\prime} \cos \theta^{\prime}=r_{1} i^{1}-a \sin \theta^{\prime \prime} \cos \lambda \cdot i^{\prime \prime} \\
e^{\prime} \sin \theta^{\prime}=x_{1} \ddot{i}^{\prime}+a i^{\prime}+a \cos \theta^{\prime \prime} \cos \lambda . i^{\prime \prime}
\end{array}\right\}
$$
and equation (8) becomes:

$$
\left.\begin{array}{l}
r_{2} \cos \theta^{\prime \prime} \cdot i^{\prime \prime}=b \sin \lambda \cdot i^{\prime}+a \sin \theta^{\prime \prime} \cdot i^{\prime \prime} \\
r_{2} \sin \theta^{\prime \prime} \cdot i^{\prime \prime}=-a \cos \lambda \cdot i^{\prime}-a \cos \theta^{\prime \prime} \cdot i^{\prime \prime}
\end{array}\right\}
$$

The torque, power-output, power-intake, power-factor, and efficiency curves of the repulsion motor plotted on speed-base $\left(f^{\prime \prime}\right)$ may be calculated from equations (9) and (10) as follows:

In the first place, $r_{1}, e^{\prime}, a$, and $x_{1}$ are known from the design of the motor and from the given value and frequency of the primary voltage.

In the second place, $\lambda$ and $r_{2}$ may be each varied at will, and therefore the curves are to be plotted for each of a series of arbitrarily assigned values of $\lambda$ and $r_{2}$. In the third place, $f^{\prime \prime}$ may be taken as the independent variable, upon which the tracing of the curves depends, and the values of torque, power-output, power-intake, etc., calculated for various assigned values of $f^{\prime \prime}$.

Thus, from an assigned value of $f^{\prime \prime}$ the value of $b$ is known. From this, together with the known values of $r_{1}, e^{\prime}, a$, and $x_{1}$, and the chosen values of $\lambda$ and $r_{2}$, the values of $i^{\prime}, i^{\prime \prime}, \theta^{\prime}$, and $\theta^{\prime \prime}$ are determined by equations (9) and (10). Then for the given speed $f^{\prime \prime}$ we have:

$$
\begin{array}{ll}
\text { power-intake } & =e^{\prime} i^{\prime} \cos \theta^{\prime} \\
\text { power-factor } & =\cos \theta^{\prime} \\
\text { power-losses } & =i^{\prime 2} r_{1}+i^{\prime \prime 2} r_{2}+\text { core losses.* }
\end{array}
$$

*Eddy current and hysteresis losses are to be calculated independently of the above ideal theory, from the known value of $\phi^{\prime}, \Phi .^{\prime \prime}, \lambda, f^{\prime}$ and $f^{\prime \prime}$ See following discussion of Mr. Steinmetz's paper. 


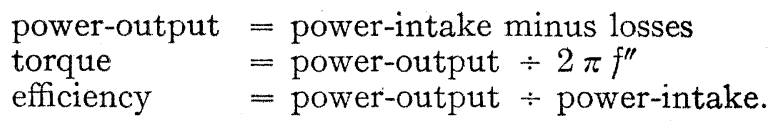

The above outline of the method of calculating the characteristic curves of the repulsion motor is sufficiently simple as a matter of theory but it is quite laborious as a matter of fact; and therefore certain of the terms of low value may be advantageously discarded in the final algebraic expressions for $i^{\prime}, \imath^{\prime \prime}, b^{\prime}$, and $\theta^{\prime \prime}$.

\section{Discussion.}

(a) It may be worth while to point out the correspondence between Mr. Steinmetz's quantities $Z$ and $x$ (see page 19 of Mr. Steinmetz's paper), and the quantities which the writer represents by $a$ and $b$, especially inasmuch as Mr. Steinmetz seems not to have specified the meaning of his symbol $x$.

Mr. Steinmetz's $Z$ is identical with $a$, and Mr. Steinmetz's $x$ (see equation (4), page 19) is equal to $f^{\prime} / f^{\prime \prime} \times b$.

When magnetic leakage is not very great, an ampere-turn, whether on the primary or secondary, produces sensibly the same amount of flux through the secondary, and in this case Mr. Steinmetz's $Z$ and $x$ are identical, if, as is assumed for the sake of simplicity, the primary and secondary have the same number of conductors.

(b) Mr. Steinmetz seems to say on page 14 that inductancereactance in the secondary of a transformer becomes capacityreactance in its effect upon the primary! The fact is that the decrease of lag of the primary current of a transformer due to load on the secondary is essentially the same effect as the decrease of lag of total current when a circuit of comparatively low inductance is connected in parallel with a highly inductive circuit.

Aside from difference in size or in design, it is not clear why Mr. Steinmetz's Fig. 3 should have a higher power-factor than his Fig. 2 (this matter is considered in detail under $c$ ) and even if Mr. Steinmetz's apparent statement regarding the relative powerfactors of Figs. 2 and 3 is correct, his explanation is not convincing.

The series motor provided with a Ryan compensating winding (c) to annul armature reaction is, when operated by alternating current, identical in its action to the repulsion motor, save in one respect, which is mentioned under $d$.

Consider, for example, Mr. Steinmetz's Fig. 5, which is equivalent to his Fig. 6. This constitutes a repulsion motor. The same structure with all three windings connected in series would be a series motor with a Ryan compensating winding. Let us consider the correspondences between the two machines on the assumption that coil resistances are zero; that magnetic leakage is zero; and that magnetic reluctance of core is zero.

Under these conditions the compensating winding $A_{2}$ and the armature winding together constitute an ideal transformer (zero 
resistance, zero magnetic leakage, and zero magnetic reluctance*) so that the current in the short-circuited armature of Fig. 5 is exactly equal ir value and opposite in phase to the main current in winding $A_{2}$, and the electromotive force induced in the armature by motion is transformed to or exactly reproduced in winding $A_{2}$, except that its phase is reversed.

Now, the reversal of phase is merely a matter of reversing connections so that the current induced in the armature is exactly the current which would flow through the armature if it were properly connected in series with the two field windings; and the electromotive force which opposes the flow of current through the two field windings $A_{1}$ and $A_{2}$ in Fig. 5 is exactly the electromotive force which would oppose the flow of current if the armature were properly connected in series with the two field windings.

Under the above-mentioned ideal conditions, the motor action of the device is exactly the same, its power-factor is the same; in fact, everything is the same, with the one exception mentioned under $d$, be the device connected as a repulsion motor or as a series motor.

So much for the ideal case; but what are the effects of coil resistances, magnetic leakage and core reluctance? These effects vitiate the motor action and they vitiate motor action more when the device is connected as a repulsion motor than when the device is connected as a series motor. This may be made clear by the following simple considerations:

When all the windings are connected in series the current is the same in all and the armature current is exactly in phase with the torque-producing flux. When the armature is short-circuited and the armature current produced by transformation from the winding $A_{2}$, then (a) the armature current (secondary) is slighty less in value than the main current (ratio of primary to secondary turns equal to unity) on account, primarily, of core reluctance; and $(b)$ the armature current differs in phase slightly from the main current and therefore from the torque-producing flux on account, primarily, of coil resistances and magnetic leakage.

Therefore, other things being equal, the torque is lessened on account of coil resistances, magnetic leakage, and core reluctance.

(d) The one difference between the compensated-series motor and the repulsion motor is the above-described transformer action and the production of cross-flux $\left(M_{2}\right.$ in Mr. Steinmetz's paper) associated therewith.

This transformer action is, however, under ideal conditions entirely independent of motor action, whereas under practical conditions this transformer action, itself incomplete, detracts from the action of the structure.

*It would be well if the "practical man" realized that once in a while, at least, the "theoretical man" sets forth ideal conditions in order that a practical matter may be made simple enough for the practical man to understand. 
When the device (Fig. 5) is connected as a compensated series. motor, no cross-flux is produced and the cross-reluctance of the core may be made high, perhaps with advantage; certainly with. saving of material.

(e) There is one point wherein the repulsion motor has an advantage over the compensated-series motor, and in regard to which the writer agrees with Mr. Steinmetz. This is in the matter of hysteresis and eddy-current losses in the armature core.

In the compensated series motor the armature is subjected to reversals of magnetization and the hysteresis and eddy-current losses are high.

In the repulsion motor the effect of the cross-flux is to produce a resultant magnetization of the armature core, which rotates at synchronous speed. This rotating magnetization is elliptical below and above synchronous speed of the armature, and circular at synchronous armature speed, so that in the repulsion motor the hysteresis loss in the armature core is large at standstill; it decreases to zero at synchronous speed, and increases again at speeds above synchronism.

The writer does not, however, agree with Mr. Steinmetz in his statements concerning the variation with speed of hysteresis loss in the armature core of the series motor. This loss is large at standstill, it decreases to about $\frac{1}{2^{11}}$ as great a value at synchronous speed and increases again at speeds above synchronism.

By B. G. Lamme.-(Communicated after Adjournment):It appears that there is a possible misunderstanding of the use of the term "straight-series" as applied by the writer to one class of single-phase motors. By this term the writer means motors in which the armature and field windings are all directly in series, and in this class is included motors with compensating windings in the poles. In the discussion of Mr. Steinmetz's paper the writer referred to the armature as being practically non-inductive and the field as being the inductive element. For best proportions such a motor would naturally be of the compensated type, and the writer's discussion was based primarily upon "straight-series" motors having compensating windings, although no direct statement to that effect was made. The series motor without compensating winding would undoubtedly have considerably higher armature self-induction. than the compensated motor, and would naturally have lower power-factor. 
[Communicated after Adjournment by Comfort A. Adams.] Note:-Vectors are indicated by bold-faced capitals.

In connection with Mr. Steinmetz's very neat and compact treatment, the following graphical representation may be of in. terest.

Assume that the reluctance of the main magnetic circuit is constant and the same in all radial directions. Reduce all secondary quantities to primary turns, and at first, neglect primary resistance and core loss; these may be taken into account later.

Referring to Fig. 1, which is a time vector diagram:

$I_{1}=$ primary current and m.m.f.

$I_{2}=$ secondary current and m.m.f.

$\mathbf{I}_{1}{ }^{\prime}=\mathbf{I}_{1} \cos \beta=$ the component of the primary m.m.f. in the secondary axis, and

$\mathbf{I}_{2}{ }^{\prime}=\mathbf{I}_{2} \cos \beta=$ the component of the secondary m.m.f. in the primary axis, where

$\beta=$ the angle between the two axes, determined by the position of the brushes.

$\mathrm{J}_{1}^{\prime}=\mathrm{I}_{1}+\mathrm{I}_{2} \cos \beta=$ the resultant m.m.f. along the primary axis;

$\Phi^{\prime}{ }_{1}=$ the corresponding flux.

$\mathbf{E}^{\prime}{ }_{1}=j x_{0} \mathrm{~J}^{\prime}{ }_{1}=$ the corresponding primary e.m.f. in quadrature with $\Phi^{\prime}{ }_{1}$, where $x_{0}$ is the exciting reactance.

$\overline{\phi_{1}^{\prime} \phi_{1}}=$ the primary leakage flux, in phase with and proportional to $I_{1}$ :

$\overline{E^{\prime}{ }_{1} E_{1}}=j I_{1} x_{1}=$ the corresponding primary drop due to the leakage reactance, $x_{1}$;

$\Phi_{1}=$ total primary flux:

Draw $\overline{J^{\prime}{ }_{1} A}$ parallel and proportional to $\overline{\phi_{1}{ }^{\prime} \phi_{1}}$, then

$\overline{O A}=\mathrm{J}_{1}$ is the current which would produce the flux $\Phi_{1}$ in the main magnetic circuit. It is the total equivalent exciting current in the primary axis.

$\mathbf{E}_{1}=j x_{0} \mathbf{J}_{1}=$ total primary inductive e.m.f., proportional to $\phi_{1}$, and in quadrature therewith.

$E_{1} E_{0}=\mathrm{I}_{1} r_{1}=$ the ohmic drop in primary, and

$\mathbf{E}_{0}=$ the impressed e.m.f. For the present $I_{1} r_{1}$ will be neglected and $E_{1}$ will be assumed equal to $E_{0}$, and constant

$\phi_{1}$ and $J_{1}$ are then constant for constant $E_{1}$.

$\mathbf{T}_{2}{ }^{\prime}=\mathbf{I}_{2}+\mathbf{I}_{1} \cos \beta=$ resultant $\mathrm{m}$.m.f. along secondary axis;

$\Phi_{2}{ }^{\prime}=$ the corresponding flux; and 
$\mathbf{E}_{2}{ }^{\prime}=-j x_{0} \mathrm{~J}_{2}{ }_{2}=$ the corresponding induced e.m.f.;

$\overline{\phi_{2}{ }^{\prime} \phi_{2}}=$ the secondary leakage flux; and

$\frac{E_{2}^{\prime} E_{2}}{E_{2}}=-j I_{2} x_{2}=$ the corresponding e.m.f.

$\Phi_{2}=$ the total secondary flux;

$\mathrm{J}_{2}=$ the equivalent exciting current.

$\mathrm{E}_{2}=-j x_{0} \mathrm{~J}_{2}=$ the corresponding e.m.f.

$\overline{O S}=\mathrm{I}_{2} r_{2}=$ secondary ohmic drop.

$\overline{S T}=j I_{2} x_{2}=\overline{E_{2} E^{\prime}}{ }_{2}=$ secondary leakage drop, where $r_{2}$

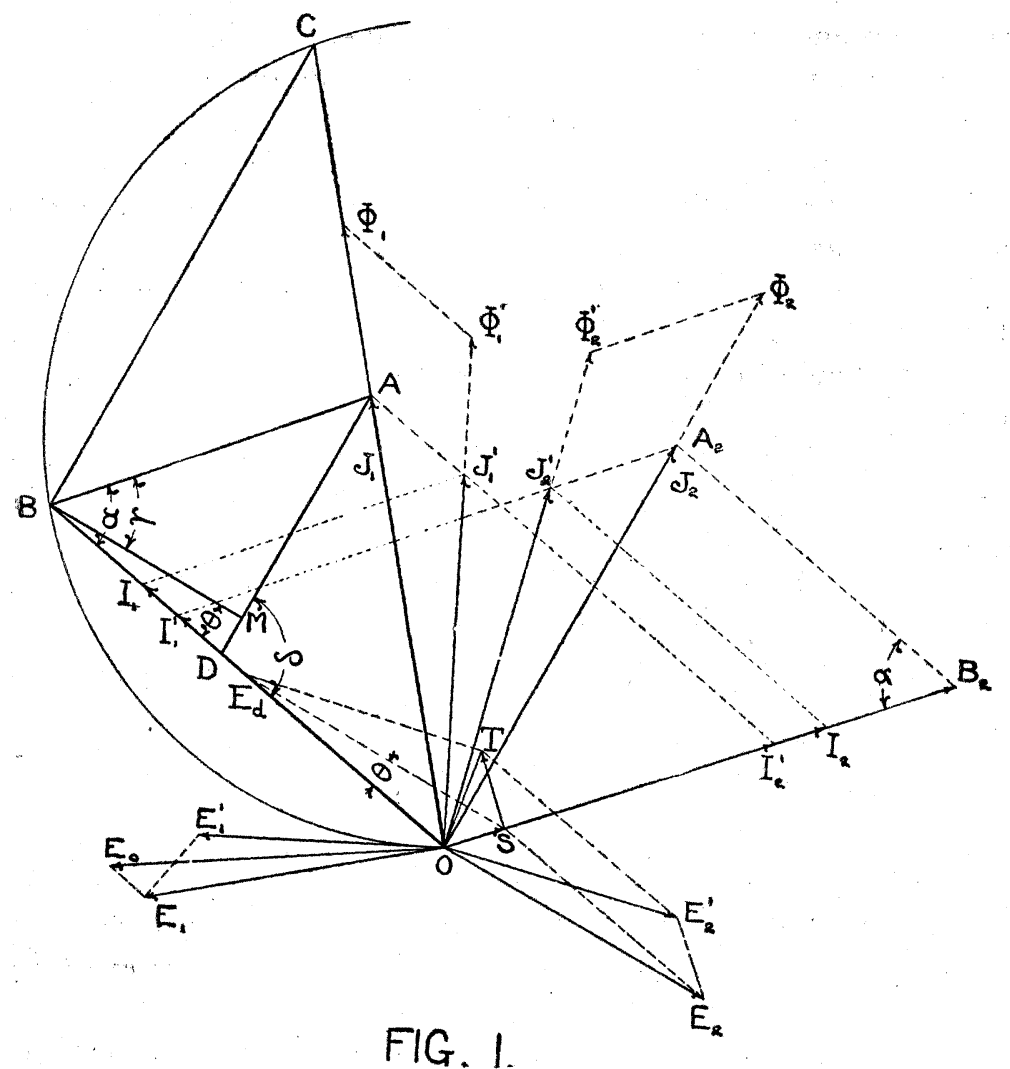

and $x_{2}$ are the secondary resistance and leakage reactance respectively.

Let $\phi_{d}=$ the dynamic flux perpendicular to the secondary axis and produced entirely by the component $I_{1} \sin \beta$, of the primary m.m.f., since the secondary m.m.f. is perpendicular to that direction and contributes nothing. Then the dynamic e.m.f. induced by the rotation of the armature through this flux will be: 
$\mathbf{E}_{a}=a x_{0} \mathbf{I}_{1} \sin \beta$, in phase with $\mathbf{I}_{1}$, where $a$ is the speed in per cent of synchronism.

Complete the parallelograms $O I^{\prime}{ }_{2} A B$ and $O I_{1}^{\prime} A_{2} B_{2}$.

$$
\begin{aligned}
& \mathbf{E}_{d}+\mathbf{E}^{\prime}{ }_{2}=\overline{O T}=\mathbf{I}_{2} \mathbf{Z}_{2} \text { or } \\
& \mathbf{E}_{d}+\mathbf{E}_{2}=\overline{O S}=\mathbf{I}_{2} r_{2} \text { and } \\
& a x_{0} \mathbf{I}_{1} \sin \beta-j x_{0} \mathbf{J}_{2}=\mathbf{I}_{2} r_{2} \\
& \text { lograms } O I^{\prime} A B \text { and } O I_{1}{ }_{2} A_{2} B_{2} .
\end{aligned}
$$

Let: $\mathcal{R}_{0}=$ reluctance of main magnetic circuit:

$\Omega_{1}=$ reluctance of primaiy leakage circuit;

$\Re_{2}=$ reluctance of secondary leakage circuit.

Then: $\Omega_{0} \equiv \frac{J^{\prime}{ }_{1}}{\phi^{\prime}{ }_{1}}=\frac{J_{1}}{\phi_{1}}=\frac{J_{{ }_{2}}{ }^{\prime}}{\phi^{\prime}{ }_{2}}=\frac{J_{2}}{\phi_{2}}=\frac{\overline{A J_{1}^{\prime}}}{\overline{\phi_{1} \phi^{\prime}{ }_{1}}}=\frac{\overline{A_{2} J^{\prime}{ }_{2}}}{\overline{\phi_{2} \phi^{\prime}{ }_{2}}}$

$$
\mathfrak{R}_{1} \equiv \frac{I_{1}}{{\phi^{\prime}{ }_{1} \phi_{1}}_{1}} \text { and } \mathfrak{R}_{2} \equiv \frac{I_{2}}{\phi^{\prime}{ }_{2} \phi_{2}}
$$

$\frac{\overline{O B}}{I_{1}}=\frac{\mathfrak{R}_{0}+R_{1}}{\mathfrak{R}_{1}}=\frac{x_{0}+x_{1}}{x_{0}}=\nu_{1}$, the primary leakage coefficient,

and

$\frac{\overline{O B_{2}}}{I_{2}}=\frac{\mathfrak{R}_{0}+R_{2}}{\mathfrak{R}_{2}}=\frac{x_{0}+x_{2}}{x_{0}}=\nu_{2}$, the secondary leakage coefficient.

Then

and

$$
\overline{O B}=\nu_{1} \mathrm{I}_{1}
$$

$$
\begin{aligned}
& \overline{O B_{2}}=\nu_{2} \mathbf{I}_{2} \\
& \mathbf{J}_{1}=\nu_{1} \mathbf{I}_{1}+\mathbf{I}_{2} \cos \beta \\
& \mathbf{J}_{2}=\nu_{2} \mathbf{I}_{2}+\mathbf{I}_{1} \cos \beta
\end{aligned}
$$

Draw $\overline{A D}$ parallel to $\mathrm{J}_{2}$ and $\overline{B M}$ perpendicular to $\overline{A D}$. In the triangle $\overline{O A D}, \overline{O A}=\mathrm{J}_{1}$ is constant, and angle $\overline{O D} A$ $=\delta=90^{\circ}+\theta$, where $\theta$ is the small angle between $\mathbf{E}_{d}$ and $\mathbf{E}_{2}$. It may be easily determined as follows: Express $I_{1}$ in terms of $\mathrm{J}_{2}$, it is:

$$
\mathrm{I}_{1}=\mathrm{J}_{2} \frac{r_{2}+j x_{0} \nu_{2}}{r_{2} \cos \beta+a x_{0} \nu_{2} \sin \beta}
$$

then $\tan \left(\mathbf{J}_{2} \mathbf{I}_{1}\right)=\cot \theta=\frac{x_{0} \nu_{2}}{r_{2}}$

and $\tan \theta=\frac{r_{2}}{\nu_{2} x_{0}}=\frac{r_{2}}{x_{0}+x_{2}}$ which is constant. 
Therefore $\left\langle\delta=90^{\circ}+\theta=\right.$ constant, and the point $\bar{D}$ will always fall on the arc of a circle through $\overline{O D A}$.

Draw $\overline{B C}$ parallel to $\overline{D A}$ and $\mathrm{J}_{2}$; then $O C / O A=O B / O D$ but $O B=\nu_{1} I_{1}$

and $\overline{O D}=\overline{O B}-B D=\nu_{1} I_{1}-\frac{I_{1} \cos ^{2} \beta}{\nu_{2}}=\nu_{1} I_{1}\left[1-\frac{\cos ^{2} \beta}{\nu_{1} \nu_{2}}\right]$

Therefore $\quad \frac{O C}{O A}=\frac{1}{1-\frac{\cos ^{2} \beta}{\nu_{1} \nu_{2}}}=\frac{1}{\sigma}$

where

$\sigma=1-\frac{\cos ^{2} \beta}{\nu_{1} \nu_{2}}$ is the leakage factor.

Then $\overline{O C}=\frac{\overline{O A}}{\sigma}=\frac{J_{1}}{\sigma}$ and is constant.

Thus, since angle $\overline{O B C}=\delta=$ constant, the locus of $\bar{B}$ is the circulararc $\overline{O B C}$ But $\overrightarrow{O B}=\nu_{1} I_{1}$ and $\overrightarrow{B A}=I_{2} \cos \beta$, i.e., proportional to $I_{1}$ and $I_{2}$ respectively. The triangle $\overline{O B A}$ thus shows clearly the relative magnitudes and phases of $I_{1}$ and $I_{2}$, for the various positions of $B$ on the arc $\overline{O B C}$.

\section{Speed.}

The different positions of $B$ correspond to different speeds and to different values of the angle $\gamma$. There is therefore some relation between the speed $a$ and the ang'e $r$. But as the latter is the angle between $E_{2}$ and $I_{2}$ its value is readily found, thus; express $\mathbf{I}_{2}$, in terms of $\mathbf{E}_{2}$; it is

$$
\mathrm{I}_{2}=\mathrm{E}_{2_{r_{2}} \cos \beta+a x_{0} \nu_{2} \sin \beta}
$$

then $\tan \left(\mathrm{E}_{2} \mathrm{I}_{2}\right)=\tan \gamma=a \tan \beta$

which is directly proportional to the speed, or

$$
a=\frac{\tan \gamma}{\tan \beta}
$$

It should be noted that the scale according to which tan $\gamma$ is a measure of the speed, depends upon the brush angle $\beta$.

There are several linear methods of representing the speed, but the angular method is more convenient for the present purpose. 
Referring to Fig. 2, extend $\overline{B M}$ until it intersects the large circle at $\overline{N ;}$ draw $\overline{N C}, \overline{N A B_{0}}$ and $\overline{O B}_{0}$. Then

$\left\langle\overline{N C O}=\left\langle\overline{N B_{0} O}=\theta\right.\right.$, being inscribed in the same arc $O N$.

$\angle \overline{N B C}=90^{\circ} \cdot . \overline{N C}$ is a diameter of circle $\overline{O B C}$.

Draw $O F$, making angle $\theta$ with $\overline{O A}$; then $b$ is the center of the circle $\overline{O B C}$.

As $B$ moves around to the right, $r$ and the speed decrease, until $\alpha=\theta$, when $\gamma$ and the speed are zero. Thus $B_{0}$ corresponds to $a=0, \widetilde{O B_{0}}$ is proportional to the primary and $\overrightarrow{B_{0} A}$ to the secondary short-circuit current.

\section{Torque.}

The dynamic power is

where

$$
P_{d}=E_{d} I_{2} \cos \alpha
$$

The torque, in synchronous watts is then,

$$
\alpha=r+\theta
$$

$$
T=(1 / a) E_{d} I_{2} \cos \alpha
$$

or substituting from equation (2)

$$
T=x_{0} I_{1} I_{2} \sin \beta \cos \alpha
$$

Draw $\overline{A H}$ perpendicular to $\overline{O B}$, then

and since

$$
\overline{H B}=I_{2} \cos \beta \cos \alpha
$$

$$
\begin{gathered}
\overline{O B}=\nu_{1} I_{1} \\
T=\overline{O B} \times \overline{H B} \frac{x_{0} \tan \beta}{\nu_{1}} *
\end{gathered}
$$

Thus the torque is proportional to the product of $\overline{O B}$ and $\overline{H B}$ for any given brush position, but the constant of proportionality varies with the brush position.

The locus of the point $H$ is the semicircle $\overline{O H A}$, since $<\overline{O H A}$ $=90^{\circ}$.

The Torque Efficiency, or torque per ampere, may be readily obtained by substituting for $I_{2}$ in eq. $14 \mathrm{a}$, its value in terms of $I_{1} ;$ thus,

* Note.-A very simple and elegant method of representing the torque by a single line, is given by $\mathrm{Dr}$. Th. Lehmann, in L'Eclairage Electrique, for February i3, 1904. 
$T=I^{2}{ }_{1} \frac{x_{0} \sin \beta \cos \beta}{\nu_{2}}\left[1-\frac{r_{2}}{r^{2}{ }_{2}+\nu^{2}{ }_{2} x_{0}{ }^{2}}\left(r_{2}+\nu_{2} x_{0} a \tan \beta\right)\right]$ or, since $r_{2}^{2}$ is ordinarily negligible with respect to $\nu_{2}{ }^{2} x_{0}{ }^{2}$

$$
T=I_{1}{ }^{2} \frac{x_{0} \sin \beta \cos \beta}{\nu_{2}}(1-a \tan \theta \tan \beta)
$$

Again, $a \tan \theta \tan \beta$ is very small at ordinary speeds and we have approximately:

$$
T=I_{1}^{2} \frac{x_{0} \sin \beta \cos \beta}{\nu_{2}}
$$

i.e., the torque is approximately proportional to the square of the current. It also appears from this last equation that the maximum torque efficiency corresponds to a brush angle $\beta=45^{\circ}$. This, however, is not the angle corresponding to the maximum torque or the highest power-factor, as will appear later:

$$
\text { Maximum Speed. }
$$

As $B$ moves io the left the torque decreases and speed increases until $\overparen{B H}$, and thus the torque vanishes at $B_{m}$, which corresponds to the maximum possible speed. At this point $\alpha=90^{\circ}$ and $\gamma=90^{\circ}-\theta$; but since $\theta$ is, under normal conditions, very small, tan $\gamma$ and thus the speed, is very large, much beyond the safe mechanical limit as well as that of commutation.

\section{POWER-FACTOR.}

The angle $\phi$ (between $E_{1}$ and $I_{1}$ ) decreases steadily from standstill to maximum speed, as $B$ moves from $B_{0}$ to $B_{m}$, and the power-factor increases accordingly, The factors which affect $\phi$ for any given speed will be considered later.

Eliminating between equations (1) (2) (3) and (6);

$$
\begin{array}{r}
\mathrm{I}_{1}=\frac{\mathrm{E}_{1}}{x_{0}} \frac{\nu_{2} x_{0}-j r_{2}}{\nu_{1} r_{2}+a x_{0} \sin \beta \cos \beta+j x_{0} \sigma \nu_{1} \nu_{2}} \\
\tan \phi=\frac{\frac{r_{2}^{2}+\sigma x_{0}^{2} \nu_{2}^{2}}{x_{0} \nu_{2}(1-\sigma)}+a r_{2} \tan \beta}{r_{2}+a x_{0} \nu_{2} \tan \beta}=\frac{\tan \theta+\frac{\sigma}{\tan \alpha}}{1-\sigma}
\end{array}
$$

As $\theta$ is normally very small, we have approximately

$$
\tan \phi=\frac{\sigma}{(1-\sigma) \tan \gamma}=\frac{\sigma}{a(1-\sigma) \tan \beta}
$$


which is inversely proportional to the speed. Mr. Steinmetz has given one explanation (page 25) why the power-factor does not in fact increase with the speed beyond a certain limit, but reaches a maximum and then falls off.

\section{Primary Power.}

$P_{1}=E_{1} I_{1} \cos \phi$, is proportional to the altitude of the triangle $O B A$, (since $E_{1}$ is assumed constant), and its maximum value occurs when $B$ is at the top of the circle $\overline{O B C}$.

The circle diagram ${ }^{1}$, Fig. 2 , is thus a great help in picturing the general relations of the several variables (currents, power-factors, speed, torque, and power), and the manner of their variation

It is also very useful in tracing the effect of changes in the constants of the motor, as will appear from what follows.

\section{Impressed. Electromotive Force.}

Since each line of the diagram is proportional to $E_{1}$, other things being equal, the currents will vary directly with $E_{1}$ and the torque and power with $E_{1}{ }^{2}$.

\section{Primary Resistance.}

If $\mathbf{E}_{1}$ is assumed constant, $\mathbf{E}_{0}\left(=\mathbf{E}_{1}+\mathbf{I}_{1} r_{1}\right)$, the real impressed e.m.f., must vary slightly; and since the tocus of the extremity of $I_{1}$ is a circle, the locus of the extremity of $I_{1} r_{1}$ is a similar circle, as indicated in Fig. 2.

But practically, $E_{0}$ would be kept constant and $E_{1}$ would vary, decreasing slightly as $I_{1}$ increases, while the whole diagram shrinks in the same proportion. Otherwise the effect of the primary resistance is to throw $E_{0}$ more nearly into phase with $I_{1}$ and thus to increase the power-factor.

\section{CORE LLSSES.}

To take account of the core losses, a small energy component must be added to the primary current, which not only increases $I_{1}$ but advances its phase and increases the power-factor.

\section{EFFECT OF VARIation OF Constants.}

Before proceeding, it will be convenient to assemble and transform a few of the equations which show the relations between the several constants and the diagram.

1. Much the same diagram has been developed by Osnos, Elektrotechnische Zeitschrift, October 29, 1903, and Blonde1, L'Eclairage Electrique, November and December, 1903, but by quite different methods, and from different starting points. 


$$
\begin{gathered}
\overline{O A}=J_{1}=\frac{E_{1}}{x_{0}} \\
\overline{O C}=\frac{\overline{O A}}{\sigma}=\frac{E_{1}}{\sigma x_{0}} \\
\sigma=1-\frac{\cos ^{2} \beta}{\nu_{1} \nu_{2}}=\frac{x_{0}^{2}\left(1-\cos ^{2} \beta\right)+x_{0}\left(x_{1}+x_{2}\right)+x_{1} x_{2}}{x_{0}{ }^{2}+x_{0}\left(x_{1}+x_{2}\right)+x_{1} x_{2}} \\
=\frac{\Omega_{1} \Omega_{2}\left(1-\cos ^{2} \beta\right)+\Omega_{0}\left(\Omega_{0}+\Omega_{1}+\Omega_{2}\right)}{\Omega_{1} \Omega_{2}+R_{0}\left(R_{0}+\Omega_{1}+\Omega_{2}\right)}
\end{gathered}
$$

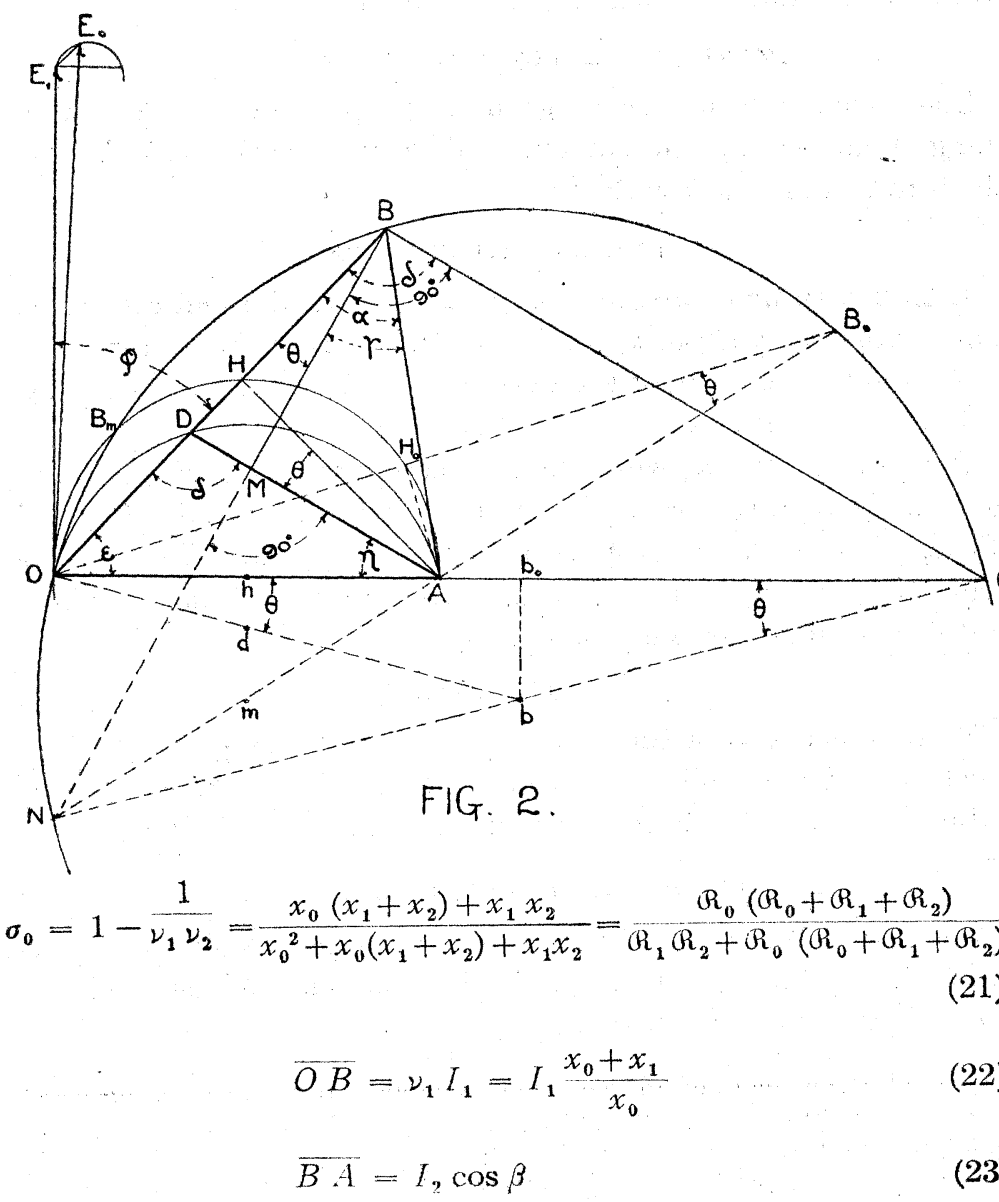




$$
\begin{gathered}
\tan \theta=\frac{r_{2}}{\nu_{2} x_{0}}=\frac{r_{2}}{x_{0}+x_{2}} \\
\tan \gamma=a \tan \beta \\
T=\overline{O B} \cdot \overline{H B} \frac{x_{0} \tan \beta}{\nu_{1}}
\end{gathered}
$$

\section{Secondary Resistance.}

A change in $r_{2}^{*}$ affects neither $\overline{O A}, \overline{O C}$, nor the relation between the speed and $\tan \gamma$, but it does alter the position of the center $b$ of the circle $\overline{O B C}$, since

$$
\overline{b_{0} b}=\frac{\overline{O C}}{2} \tan \theta=r_{2} \frac{E_{1}}{\sigma \nu_{2} x_{0}^{2}}
$$

i.e., an increase in $r_{2}$ means a proportional dropping down of $b$ from $\overline{O C}$, and when $r_{2}=0, b$ lies at $b_{0}$, the circle always passing through $O$ and $C$. In Fig. 3, several resistance circles are drawn, corresponding to resistances from zero to seven.

Consider the movement of $B$ corresponding to a change of $r_{2}$ under constant speed, i.e., with $\gamma$ constant.

Then $\angle \overline{A B C}=90^{\circ}-\gamma$, and is constant, and $B$ will move on a circular arc $\overline{A B C}$, whose center lies on the perpendicular erected at the centre of $A$.

Imagine $B$ to move along one of these constant-speed circles until $\overline{B A C}=90^{\circ}$, then $\overline{B C}$ will be a diameter of circle $\overparen{B A C}$, and the intersection of $\overline{B C}$ with the perpendicular $\overline{m_{0} m}$ will be the center of the constant speed circle in question. Moreover, since $\overline{A B C}=90^{\circ}-\gamma,<\overline{A C m}=\gamma$. Thus, $\overline{m_{0} m}$ is directly proportional to the speed $a$ corresponding to the circle in question.

$$
\overline{m_{0} m}=\overline{m_{0} C} \tan \gamma=J_{1} \frac{1-\sigma}{2 \sigma} a \tan \beta=\frac{E_{1}}{2 x_{0}} \frac{a \tan \beta}{\frac{\nu_{1} \nu_{2}}{\cos ^{2} \beta}-1}
$$

When $a=0$, the center falls to $m_{0}$. Several constant speed circles are shown in Fig. 3.

If $B$ falls within the semicircle $\overline{O A}$, the torque is negative, if it falls within the semicircle $\overline{A C}$, the speed is negative, and points outside of semicircle $\overline{O C}$ correspond to negative values of $r_{2}$.

*It is understood that $r_{2}$ includes the total secondary resistance, inetrnal and external. 
Therefore, it is evident that for operation as motor, $B$ must fall within the area enclosed by these three semicircles.

The variation of torque and power-factor with speed and secondary resistance can be readily traced on diagram, Fig. 3.

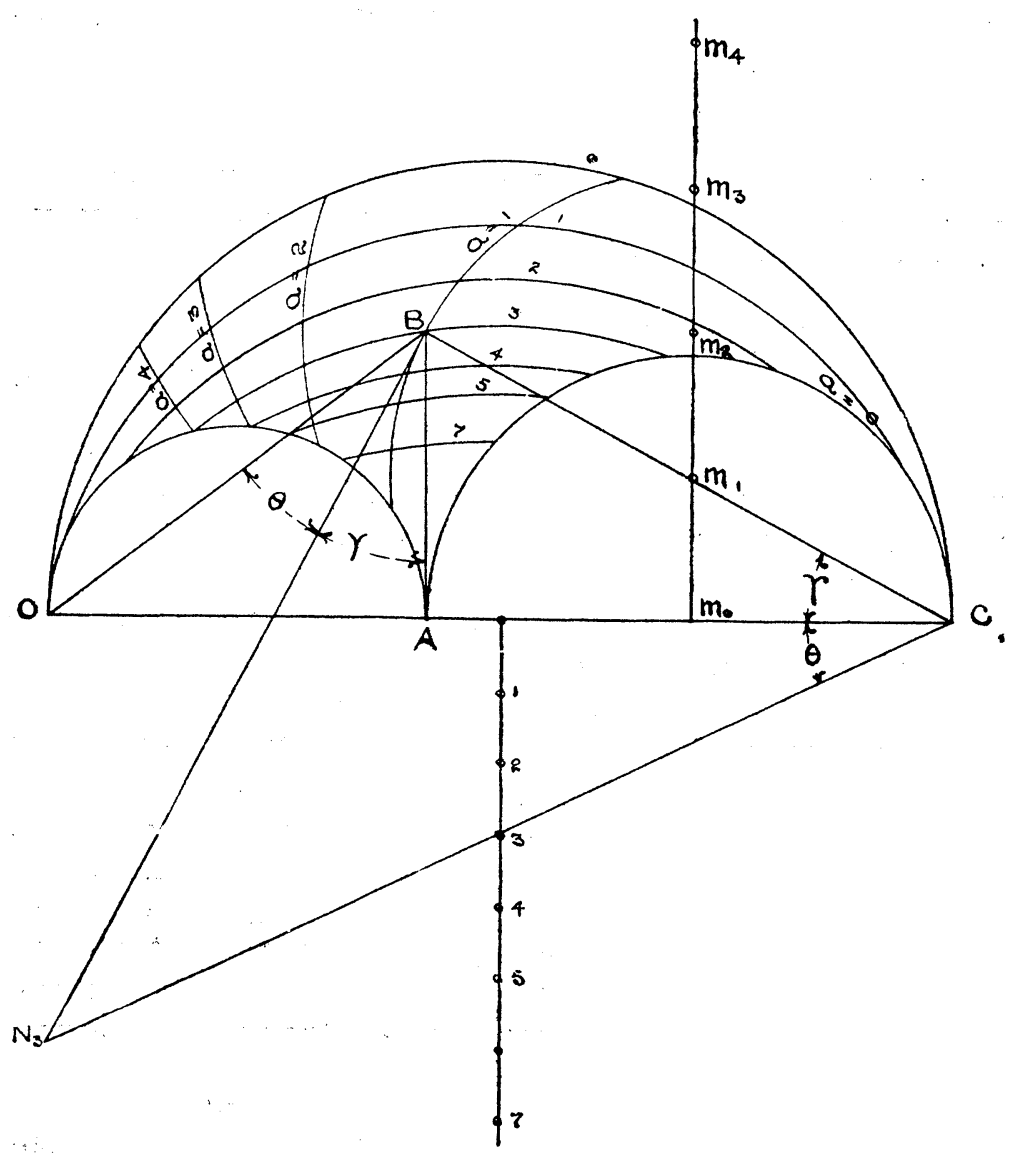

FIG. 3 .

\section{Leakage Reactance.}

Leakage in primary and secondary have for the most part the same general effect, except that $\nu_{1}$ alone effects the scale according to which $\overline{O B}$ represents $I_{1}$ (eq. 22), and also the torque scale (eq. 26); while $\nu_{2}$ alone effects $\tan \theta$ (eq. 24). But $\nu_{1}$ is constant in any given machine, varying only slightly in different types, 
whereas $\nu_{2}$ which includes any external reactance connected between the brushes, may have a wide variation.

Consider then, a variation in $\nu_{2}$ or $x_{2}$. Assume that there is no external resistance between brushes, then $r_{2}$ may be neglected without seriously affecting the general results of the analysis.

Referring to the equations of page 70 , a change in $\nu_{2}$ or $x_{2}$ does not affect $\overline{O A}$, it does affect $\sigma$ and thus $\overline{O C}$. In Fig. 4, several $\overline{O B C}$ circles are drawn, corresponding to values of $x_{2}$, varying from +4 to $-4,0.4$ being the normal value for the machine in question.

As $x_{2}$ decreases, passes through zero and increases negatively, $\sigma$ decreases and passes through zero, and the point $C$ moves out to infinity on the right and comes back on the left.

As $r_{2}$ was neglected, $\angle \overline{O B A}=\gamma$, and if the speed be maintained constant while $x_{2}$ varies, the locus of $B$ will be a circle $\overline{O A B}$ whose center lies on the perpendicular at the center of $O A$. If $q_{1}$ is the center of the constant speed circle corresponding to $a=1$, then the $\left\langle\overline{O q_{1} A}=\right.$ twice the $\left\langle\overline{O B_{1} A}\right.$ and $\overline{O q_{1} p}=r_{1}$. Then,

$$
\begin{aligned}
& \frac{\overline{O p}}{\overline{p q_{1}}}=\tan \gamma_{1}=a_{1} \tan \beta \\
& \overline{p q_{1}}=\frac{\overline{O p}}{a_{1} \tan \beta}=\frac{E_{1}}{2 a_{1} x_{0} \tan \beta}
\end{aligned}
$$

and

The effect of the secondary resistance will be to flatten the constant-speed circles slightly.

\section{Air-Gap.}

An increase of air-gap means an increase in $R_{0}$, a decrease in $x_{0}$, and an increase in $O A$. An inspection of equation (20) shows that since $\left(1-\cos ^{2} \beta\right)$ is ordinarily a small fraction, and since $\mathfrak{R}_{1} \boldsymbol{R}_{2}$ is large as compared with $\Omega_{0}\left(\Omega_{0}+R_{1}+R_{2}\right), \sigma$ falls only a little short of being proportional to $\Omega_{0}$. Therefore, $\overline{O C}(=\overline{O A}$ $\div \sigma$ ) is nearly independent of the air-gap.

The air-gap also affects the primary-current scale slightly (eq. 22), the torque scale to a greater extent (eq. 26), and $\tan \theta$ (eq. 24).

It is seen from (eq. 16) that the current corresponding to any given torque is otherwise approximately proportional to $\sqrt{\frac{1}{x_{0}}}$, or 
to $\sqrt{R_{0}} ;$ thus, to double the gap would add about $40 \%$ to $I_{1}$, and produce a considerable decrease in the power-factor. This is easily traced on the diagram by imagining $\overline{O A}$ to increase, without affecting $\overline{O C}$.

\section{Brush Position.}

Here, again, it is desirable to neglect $r_{2}$ in order to get a simpler view of the effect of changing $\beta$.

$\overline{O A}$ is unaffected, but $O \bar{C}$ increases with decreasing $\beta$.

The secondary-current scale (eq. 23), the speed scale (eq. 25), the torque scale (eq. 26) and the torque efficiency (eq. 16) are also dependent upon $\beta$.

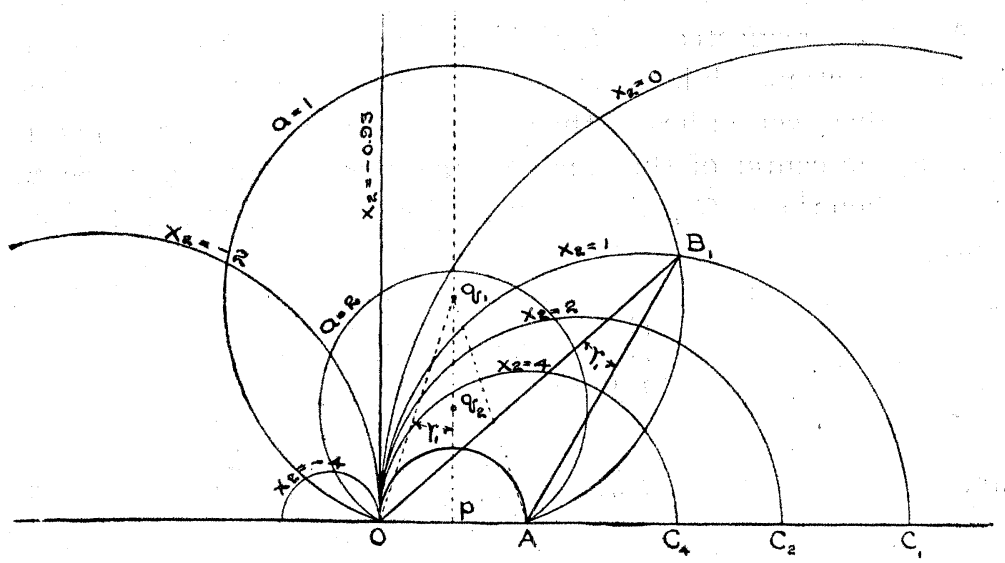

FIC. 4 .

The locus of the point $B$ with varying $\beta$ and constant speed, is not a simple curve, but has been drawn in on Fig. 5 for several speeds. Circles are also shown for values of $\beta$ varying from $0^{\circ}$ to $90^{\circ}$.

Since all losses have been neglected, the input is equal to the output, and for any given speed the altitude of the point $B$ is proportional to the torque; i.e., if $B$ moves along one of the constant-speed curves, the maximum torque for that speed corresponds to the highest point of the speed curve under consideration.

It will be observed that this maximum torque occurs with a very small brush angle $\beta$ for high speeds, decreasing to $45^{\circ}$ at standstill. This maximum torque and maximum output are obtained at the expense of a low power-factor and excessive 
current, and are far beyond normal operation, except at reduced voltage. It is somewhat analogous to the maximum output obtained from a direct-current motor by weakening its field until the counter e.m.f. equals one-half of the impressed e.m.f.

The best value of $\beta$ is that which gives the highest power factor; it is determined as follows, from (eq. 17a):

$$
\tan \phi=\frac{\nu_{1} \nu_{2}-\cos ^{2} \beta}{a \sin \beta \cos \beta}
$$

\section{FIG. 5.}

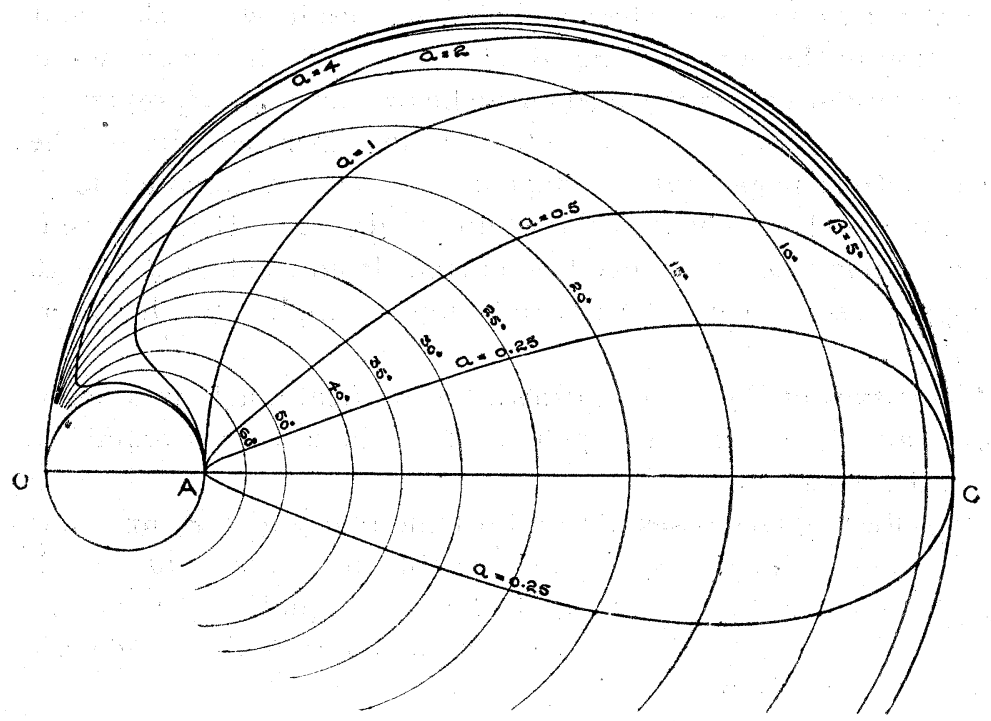

Placing the derivative of $\tan \phi$ with respect to $\cos \beta$ equal to zero and solving

$$
\cos \beta=\sqrt{\frac{1}{1+\sigma_{0}}}
$$

which is independent of the speed.

Substituting this in (30) gives for the minimum value of $\tan \phi$

$$
\tan \phi_{m}=\frac{2 \sqrt{\sigma_{0}}}{a} \nu_{1} \nu_{2}
$$

$\left.\begin{array}{l}\text { If } \nu_{1}=\nu_{2}=1.1, \quad \beta_{m}=23^{\circ} \text { and } \cos \phi_{m}=.703 \\ \text { If } \nu_{1}=\nu_{2}=1.05, \quad \beta_{m}=17^{\circ} \text { and } \cos \phi_{m}=.803\end{array}\right\}$ for $a=1$ 
The less the leakage the smaller the angle $\beta_{m}$ for maximum power-factor. Eq. (32) shows clearly the factors upon which the maximum power-factor depends.

Figs. 3,4 , and 5 together with the accompanying equations, give a fairly clear picture of the effect produced upon the operation of the repulsion motor by the variation of its several constants.

\section{Generator.}

Fig. 5 lends itself readily to the tracing of the generator action: of the repulsion motor.

While the machine is running at any constant speed as motor on constant-pressure circuit, connect it to a prime mover running at the same speed, then shift the brushes forward, the point $B$ moving to the left, reaching $A$ when $\beta=90^{\circ}$, then moving out along a similar constant-speed curve below the axis, $P_{1}$ reversing sign as $B$ crosses the axis, $\overrightarrow{O C}$. Under these conditions the power output as generator is dependent upon the brush angle, $\beta$, and the speed at which the machine is driven. It is not selfexciting, but receives its exciting current from the line, although at high speeds a part of the excitation is supplied by the rotorcurrent.

The curves of Fig. 5 were drawn for an ideal machine without. losses, and the motor and generator portions are symmetrical in consequence.

The effect of the losses is to retard the reversal of $P_{1}$ until the brush angle has attained an appreciable negative value, such that the mechanical power supplied is just equal to the losses. This effect is readily shown on the circle diagram at the expense. of extreme simplicity. 


\section{[COMMUNICATED AFTER ADJOURNMENT:]}

Discussing Papers by Slichter, Steinmetz, and Others Relating to Alternating-Current Railway Motors.

Dugald C. Jackson:- The writer notices that Mr. Steinmetz attributes the modern form of repulsion motor to Professor Elihu Thomson, and others including Mr. Blanck (whose discussions are stimulated by the papers of Messrs. Slichter and Steinmetz) attribute its development to Mr. Steinmetz. It seems only proper to point out that neither of these designations is historically. correct. The repulsion motor capable of producing continuous rotation which was created by Professor Elihu Thomson contained an armature with individual open coils. This armature was caused to rotate by the repulsion brought to bear upon the coil which at any instant chanced to be in the most effective position,- - this particular coil being short-circuited when in that position. A characteristic feature of the Thomson structure lies in the fact that the coils approach the most effective position with open circuit. When a coil reaches the effective position, it is shortcircuited by the brushes and produces torque, for the short period of the short-circuit only, as the coil recedes from its most effective position.

The device which has now come to be known by the name of repulsion motor (and which is referred to in the above-named papers) is distinctly different from this. The armature winding is a closed-coil winding and the whole of the armature winding may be traversed by the induced current at every instant. This device was invented by Anthony, Jackson, and Ryan, in 1887, and the earliest published description of such a motor with which the writer is acquainted is contained in the AnthonyJackson-Ryan patent No. 389352 which was issued on Sept. 11,1888 . Others are said to have independently invented the same device, including William Stanley and the late Charles J. Van Depoele in this country.

This type of motor has been constructed for a number of years by the Wagner Electric Mfg. Company,-which company uses the repulsion arrangement in the starting of their singlephase induction motors. Those who have had to do with these machines have long known that the repulsion motors have characteristics which afford excellent reasons for their introduction into electric railway service when the extension of electric railway lines produces a sufficient demand for an alternatingcurrent railway motor. When used in such service it is desirable to introduce into their construction special means for reducing or abating the sparking at the commutator which is common to alternating-current motors constructed with a commutator. 